\title{
En la España Medieval
}

ISSN: 0214-3038

http://dx.doi.org/10.5209/ELEM.60008

\section{La orientación de las iglesias mozárabes}

\author{
Juan Pérez Valcárcel ${ }^{1}$; Victoria Pérez Palmero ${ }^{2}$
}

Recibido: 07 de noviembre de 2017 / Aceptado: 11 de febrero de 2018

Resumen. Las iglesias mozárabes plantean numerosos puntos dudosos, empezando por el propio concepto de mozárabe, muy discutido por los especialistas. Uno de ellos es su orientación, que tiene algunas características propias que lo diferencian de otros edificios prerrománicos. Mantienen la tendencia general de la orientación hacia la salida del sol, pero presentan unas dispersiones mayores que en otros ejemplos estudiados. Existe un porcentaje significativo de iglesias cuyas orientaciones no corresponden con ninguna posición posible del orto solar e incluso existe un caso especialmente extraño, como es la ermita de Hérmedes de Cerrato, con una orientación prácticamente a norte, excepcional en las iglesias altomedievales europeas. Dada la escasez de estudios específicos sobre la orientación de las iglesias mozárabes, los autores pretenden analizar las orientaciones medidas sobre un conjunto de 49 iglesias españolas y portuguesas. Son casi los únicos lugares donde se realizó esta arquitectura, a excepción de algunos ejemplos en la Septimania francesa, cuya consideración presenta dudas y que no se incluyen en la base de datos. Se trata de definir el día que fueron orientadas y las razones simbólicas, litúrgicas o funcionales de tal orientación. Para ello se hará una breve exposición de los cálculos astronómicos necesarios. También se analizarán algunos casos atípicos de interés, muy notables en este tipo de iglesias. Palabras clave: Arquitectura mozárabe; orientación de las iglesias; orto solar; medida de la orientación; orientaciones simbólicas; orientaciones funcionales.

\section{[en] The Orientation of the Mozarabic Churches}

\begin{abstract}
The Mozarabic churches raise a lot of dubious points, beginning with the own concept of Mozarabic, much discussed by the specialists. One of them is its orientation, which has some characteristics that differentiate it from other pre-Romanesque buildings. They maintain the general tendency of the orientation towards the sunrise, but they present greater dispersions than in other studied examples. There are a significant percentage of churches whose orientations do not correspond to any possible position of the solar ortho even an amazing case, such as the hermitage of Hérmedes of Cerrato, practically to the north, exceptional in the European early-medieval churches. Given the scarcity of specific studies on the orientation of the Mozarabic churches, the authors intend to analyze the orientations measured on a set of 49 Spanish and Portuguese churches. They are almost the only places where this architecture was built, except for some examples in the French Septimania, whose consideration presents doubts and that are not included in the database. It is about defining the day that they were oriented and the symbolic, liturgical or functional reasons of such orientation. For this purpose, a brief description of the necessary astronomical calculations will be made. We will also analyze some atypical cases of interest, very remarkable in this type of churches.
\end{abstract}

Keywords: Mozarabic Architecture; Orientation of Churches; Solar Ortho; Orientation Measurement; Symbolic Orientations; Functional Orientations.

\footnotetext{
1 Universidade da Coruña

valcarce@udc.es

2 Universidade da Coruña

sas_leonidas@yahoo.es
} 
Sumario. 1. Introducción 2. La arquitectura mozárabe. 3. Medición de la orientación y factores que intervienen. 4. Orientación de las iglesias mozárabes. 4.1. Análisis de los criterios de orientación de las iglesias mozárabes. 4.2. Las iglesias del Serrablo. 4.3. Orientaciones atípicas. 5. Conclusiones. 6. Bibliografía.

Cómo citar: Pérez Valcárcel, J.; Pérez Palmero, V. (2018) La orientación de las iglesias mozárabes, en En la España Medieval 41, 171-197.

\section{Introducción}

Desde los primeros tiempos del cristianismo aparecen diversos testimonios de la costumbre de que la comunidad de fieles orase en dirección al sol y específicamente en la dirección del orto solar. Esta pretensión, que en principio no se tradujo en edificios concretos, dada la situación de clandestinidad de los cristianos, se empieza a considerar en las basílicas específicamente cristianas a partir del siglo IV. En un primer momento, algunas de las basílicas constantinianas se orientan al oeste, pero, tras el Concilio de Nicea, las iglesias cristianas se van a orientar a levante y, con algunas excepciones, esta será la regla común hasta el siglo XIII ${ }^{3}$.

Pero esto supone un problema. Una posible orientación al sur puede fijarse siempre sin ambigüedad, pero la posición del orto solar no es fija, sino que varía a lo largo del año. Esta variación depende de la latitud y tiene una amplitud notable, que en la Península Ibérica puede ser superior a $60^{\circ}$. Esto quiere decir que podemos encontrar orientaciones entre algo menos de $60^{\circ}$ y algo más de $120^{\circ}$, medidos desde el norte, que correspondan a posiciones posibles del orto solar. Por ello consideramos evidente que las orientaciones de las iglesias se fijaron un día concreto y específico para cada iglesia, puesto que su orientación marca una posición del orto que corresponde a un día concreto y sus valores presentan grandes diferencias, lo que hace que esos días se repartan a lo largo del año. La fijación de ese día y las razones por las que fue elegido ese y no otro, se tratarán en este artículo para el caso concreto de las iglesias mozárabes.

Para ello, es necesario determinar con precisión el punto del horizonte que corresponde al orto solar en cada día del año y en su caso las posibles variaciones seculares. Además es necesario corregir la posición del orto solar cuando el horizonte está en pendiente. Por último es preciso poder determinar la posible correlación de ese día en concreto con el templo, sea por su advocación o por cualquier otra circunstancia.

3 Vogel, "Sol aequinoctialis". En este artículo se hace un completo estudio de las orientaciones de los primeros templos cristianos, su simbología y su relación con la liturgia. También Delgado Gómez, "El porqué de la orientación de las iglesias" o McCluskey, "Orientation of Christian Churches". 


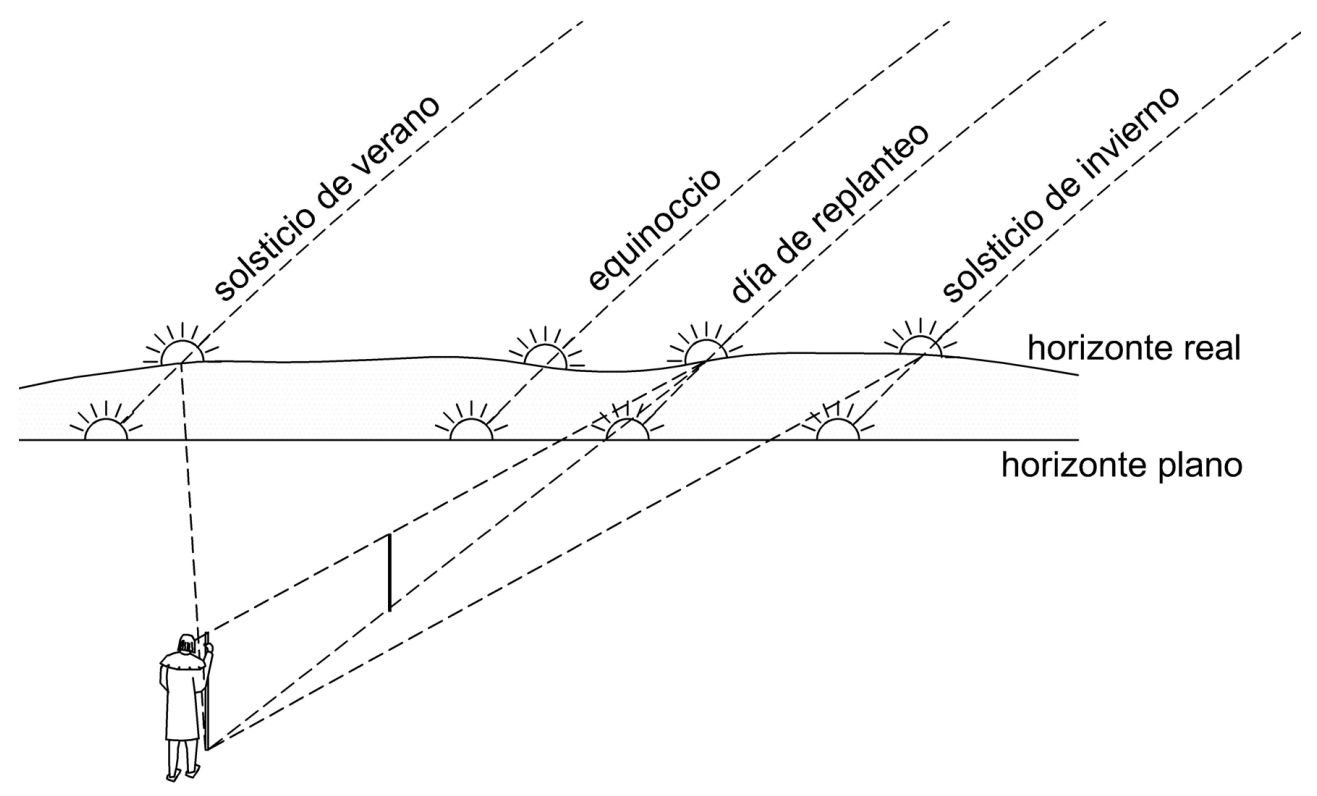

Figura 1. Variaciones anuales del orto solar y fijación de la orientación.

Para determinar la posición exacta del orto solar, considerando la altura del horizonte, hemos desarrollado un programa de ordenador, ORIENT 2.0, que permite obtener esos datos con una precisión de \pm 1 día en los equinoccios y \pm 4 días en los solsticios, que estimamos suficiente. La razón de esta diferencia de precisión es que el desplazamiento de la posición del orto solar es mucho más rápido en los equinoccios que en los solsticios -en latín solstitium (sol sistere) significa Sol detenido. Los instrumentos usados dan la misma precisión de medida en cualquier día del año, pero en el solsticio un pequeño error en la medición puede significar varios días en el resultado, al contrario que en los equinoccios. Los cálculos astronómicos pueden ser muy precisos, pero determinar las razones y circunstancias del día concreto en que se orientó la iglesia es un problema histórico bastante complejo, que exige un estudio a fondo de los calendarios medievales y que siempre presenta grandes incertidumbres.

Comparando los datos tomados con dichos calendarios podemos determinar si la orientación se hizo un día predeterminado con una intención religiosa o bien se realizó sin un motivo concreto o por las propias necesidades constructivas. Ese es el objetivo principal de este artículo. Aunque pocos, existen algunos estudios previos que vamos a referenciar para situar el problema en su contexto. No hemos encontrado ninguno dedicado específicamente al tema concreto de las iglesias mozárabes, pero hay algunos interesantes trabajos sobre el prerrománico y románico que nos proporcionan metodologías de estudio y algunas pautas que podemos comparar con nuestras propias observaciones. Una obra de referencia, pese a su antigüedad, es la 
de Nissen ${ }^{4}$, en la que se hace un completo estudio de las orientaciones de templos griegos y romanos, terminando con un amplio capítulo dedicado al cristianismo. Se le suele atribuir la hipótesis que llamaremos simbólica: supone que el día elegido fue aquel de la festividad del santo patrón de la iglesia. En nuestra opinión, esta atribución no es correcta en lo referente a los templos cristianos. Nissen estima que puede haberse aplicado este criterio simbólico, pero solo lo menciona en el caso de algunos templos griegos.

En realidad, esta hipótesis se plantea en 1822 por parte de un poeta romántico inglés, William Wordsworth, ligado a la masonería. Según Ali y Cunich, se cita esa hipótesis en la introducción a su Miscellaneous Poem XIII, en la que el poeta afirma:

Our churches, invaribly perhaps, stand east and west, but why is by few persons exactly known; not that de degree of deviation from due east often noticeable in the ancient ones was determined, in each particular case, by the point of the horizon at wich the sun rose upon the day of the saint to whom the church was dedicated $^{5}$.

Puesto que es una hipótesis sin duda atractiva, la mayor parte de los estudios realizados la analizan. Podemos citar los trabajos de investigadores ingleses que han analizado las orientaciones de templos románicos de Inglaterra y Gales. Algunos, como Cave, consideran de que la orientación simbólica debe ser rechazada en todos los $\operatorname{casos}^{6}$. Otros como Ali y Cunich estiman que las correlaciones que han encontrado en su zona de estudio pueden ser aceptables 7 . También Spinazzè afirma haber encontrado correlaciones significativas en el norte de Italia ${ }^{8}$. Pueden ser citados también algunos investigadores que han analizado diversos casos de iglesias centroeuropeas como Guszik, Conrad y Mertens, Eckstein, Büll y Hörning, o Čaval. En nuestra opinión, y sin discutir los datos aportados, creemos que la correlación estadística que aportan estos autores no es, en modo alguno, concluyente y, en todo caso, no parece extrapolable al caso estudiado. Tal vez la obra más completa en este sentido es la tesis doctoral de Hinton, que, con una metodología minuciosa de toma de datos de orientaciones y pendientes, llega a las mismas conclusiones que Cave, rechazando igualmente la hipótesis simbólica ${ }^{9}$. En todo caso, estas consideraciones no pueden ser extrapoladas a una realidad diferente ni en el espacio ni en el tiempo, como es la arquitectura mozárabe, que además suscita una enorme cantidad de dudas.

Otro aspecto que puede ser de interés, es el de las posibles interacciones con el entorno que pueden influenciar la orientación. Es un aspecto que han analizado algunos investigadores como Sassin Allen o Knapp y Ashmore, que se refieren a él como criterio paisajístico ${ }^{10}$. Nosotros preferimos definirlo como criterio topográfico, al entender que nos referimos a accidentes geográficos permanentes, mientras que el paisaje puede incluir elementos efímeros, pero es evidente que se trata una cuestión subjetiva. En el caso de las iglesias mozárabes se producen algunas notables

\footnotetext{
Nissen, Orientation, Studien zur Geschichte der Religion.

Ali y Cunich, "The Orientation of Churches: Some New Evidence", p 155.

Cave, "The Orientation of Churches".

Ali y Cunich, "The Orientation of Churches: Some New Evidence".

Spinazzè, "The Alignment of Medieval Churches in Northern-central Italy".

Hinton, Aspects of the Alignment and Location of Medieval Rural Churches.

Sassin Allen, "Church Orientation in the Landscape"; Knapp y Ashmore, "Archaeological Landscapes".
} 
situaciones, que pueden explicar algunos casos atípicos, por lo que entendemos que debe ser analizado. A título casi anecdótico, podemos citar a algunos investigadores nórdicos como Abrahamsen o Persson, que proponen una teoría basada en la determinación de la orientación por medio de un posible uso primitivo de la brújula ${ }^{11}$. No negamos su viabilidad en los casos estudiados del románico danés o sueco, pero sin duda no tiene el menor sentido en nuestro campo, por lo que hemos considerado innecesario estudiarla.

Hay muy pocos trabajos que analicen las orientaciones de iglesias prerrománicas. Uno muy destacable es el de Hoare y Sweet referido a las iglesias prerrománicas en Inglaterra, pero, a pesar de su interés, tampoco puede considerarse una fuente válida, salvo para los aspectos metodológicos, puesto que la realidad histórica es muy diferente $^{12}$. Recientemente González-García y Belmonte han publicado un artículo de gran interés sobre las orientaciones de las iglesias prerrománicas de la Península Ibérica, en el que incluyen numerosas iglesias mozárabes ${ }^{13}$. Es muy estimable la base de datos que aporta, pero entendemos que las conclusiones a las que llega son, en varios aspectos, discrepantes con nuestros propios datos, tanto por la base de datos empleada, con una serie de iglesias que estimamos que no deben ser incluidas, como por la metodología estadística. En este sentido, nuestro enfoque aporta otro punto de vista.

De acuerdo con los distintos planteamientos que hemos indicado, es posible definir varios criterios de orientación:

- Criterio simbólico. Consiste en suponer que la iglesia se orientó el día de la festividad del santo al que iba a ser dedicada. Esta hipótesis se atribuye a Nissen, pero pocos investigadores la defienden en la actualidad, tal como se ha indicado. También podemos considerar como criterios simbólicos algunos ligados a fechas concretas y destacadas, como el 1 de mayo que cita Hoare o el 25 de julio que citan GonzálezGarcía y Belmonte.

- Criterio topográfico. Se considera este criterio en el sentido fijado en el DRAE como: "Conjunto de particularidades que presenta un terreno en su configuración superficial"14. Consiste en suponer que la orientación de la iglesia está influenciada por el entorno, ya porque esté ligada a un accidente geográfico, un lugar preciso o incluso un paisaje característico. Es una hipótesis que han planteado algunos autores ingleses, como Sassin Allen o Knaps, y que puede explicar diversos casos por las características físicas o simbólicas del lugar, como se verá.

- Criterio litúrgico. Consiste en suponer que las iglesias se orientaron en la festividad de Pascua del año de su construcción. Esta hipótesis es sostenida por algunos investigadores, como Romano (1993) o McClusckey (1998), pero no se ha aplicado a la mayoría de los estudios realizados. Nosotros hemos detectado algunas notables coincidencias que nos indican que merece un análisis más completo, pese a sus notables dificultades de demostración. Entendemos importante aclarar la diferencia entre el criterio simbólico y el litúrgico o pascual, puesto que podría haber confusiones terminológicas. El criterio simbólico apunta a una fecha concreta y relacionada específicamente con el templo, que además puede ser demostrada, al repetirse la alineación en la misma fecha todos los años. En cambio el criterio litúrgico se refiere a

\footnotetext{
Abrahamsen, "Church Orientation by Magnetic Compass"; Persson, "The Orientation of Vendel Church".

Hoare y Sweet, "The Orientation of Early Medieval Churches in England”, pp 162-173.

González-García y Belmonte, "The Orientation of Pre-Romanesque Churches in the Iberian Peninsula".

Los autores ingleses suelen emplear la palabra landscape, que consideramos reduccionista y menos precisa.
} 
una festividad universal y desligada del edificio concreto y que es diferente de año en año. No puede ser demostrado, salvo conociendo la fecha exacta de replanteo. Aunque pueden existir discrepancias, entendemos que lo más oportuno es diferenciar ambos criterios y analizarlos por separado.

- Criterio constructivo. Consiste en suponer que la iglesia fue orientada en el día de su replanteo, que estaba fijado por unas adecuadas condiciones constructivas y que por tanto no dependía de ninguna festividad religiosa. Es el criterio que sostienen la mayor parte de los investigadores, aunque con alguna discrepancia que señalaremos.

En el presente artículo se analizarán cada uno de estos criterios en lo referente a las iglesias mozárabes. Se trata de determinar el criterio que puede explicar un mayor número de casos, las razones para ello y las posibles excepciones.

\section{La arquitectura mozárabe}

Puesto que el objeto de este artículo es la orientación de las iglesias mozárabes, es necesario hacer algunas consideraciones previas sobre las mismas. En primer lugar, hemos de señalar que incluso el propio adjetivo mozárabe está sujeto a fuerte controversia. Hablando con precisión, el arte mozárabe (del árabe musta 'rab que significa arabizado), solo puede aplicarse al arte de los cristianos que vivían en zonas musulmanas. La muy relativa tolerancia islámica permitía el culto cristiano en condiciones limitadas, pero imponía grandes dificultades a la construcción de nuevas iglesias. Podemos citar entre las que se han conservado en lo que, en el siglo X, era territorio musulmán, la iglesia de Melque, pese al debate que existe sobre su datación. También algunos restos en distintas iglesias de Toledo, que no podemos considerar a efectos de medida de orientaciones, puesto que no estamos seguros de si las actuales iglesias mudéjares respetaron o no su trazado original. En realidad, solo podemos hablar con propiedad de iglesias mozárabes en un único caso: las iglesias de Bobastro, construidas durante la sublevación de Omar Ben Hafsún contra el emirato de Córdoba ${ }^{15}$. A este caso se podría añadir la ermita de Santa María de la Cabeza, próxima a Bobastro, en Ronda. Es de probable origen mozárabe y se le atribuye una antigüedad similar. Sin embargo, al tratarse de una iglesia rupestre, su orientación está forzada por las características topográficas de su entorno ${ }^{16}$. Tras sofocar esta sublevación en 928, Abderramán III se proclamó califa en 929. La ya escasa tolerancia religiosa fue disminuyendo, al tiempo que los reinos cristianos del norte llevaban la frontera a la línea del Duero, quedando una amplia zona necesitada de repoblación. Diversas comunidades cristianas mozárabes emigraron hacia el norte y se establecieron en esa zona, donde edificaron sus iglesias.

En 1919, Manuel Gómez-Moreno publica su obra Iglesias mozárabes. Arte español de los siglos IX a XI (Madrid: Centro de Estudios Históricos), en la que atribuye a esas

15 A la basílica de Bobastro, ya referenciada desde 1923, se añade una nueva iglesia descubierta en 2001 (Martínez Enamorado, "Sobre las 'cuidadas iglesias' de Ibn Hafsun"), de la que no aporta ningún dato de orientación, salvo una flecha imprecisa en la figura 2 de su artículo. Los autores solo han estado en una ocasión en Bobastro, precisamente en 2004, y, en aquel momento, no se pudo visitar ni medir la segunda iglesia de Bobastro, motivo por el que no se incluye en la base de datos.

16 Medidas tomadas en distintas iglesias rupestres, desde las de la cordillera Cantábrica a las de Capadocia, muestran que la orientación está lógicamente fijada por los condicionantes del lugar. En algunas ocasiones, como en San Pedro de Rocas en Ourense o San Juan de la Peña en Huesca, el eje puede definirse por el oeste, pero no es la regla general. En muchas iglesias rupestres no es posible definir el eje con criterios solares, puesto que su entrada es lateral. 
comunidades y a su bagaje cultural la autoría de las iglesias que se construyeron en los reinos cristianos entre el final del siglo IX y comienzos del siglo XI. Pese a su evidente reduccionismo, esta hipótesis fue aceptada ampliamente. Sin embargo historiadores más recientes como Isidro Bango Torviso, la han cuestionado con sólidas razones e incluso proponen sustituir la denominación de arte mozárabe por la de arte de repoblación ${ }^{17}$, probablemente más preciso en términos históricos.

En nuestro caso, emplearemos la denominación de iglesias mozárabes por ser más común y fácilmente identificable, pero nos limitaremos a aplicarla a aquellos edificios construidos en esas fechas, con los rasgos comúnmente admitidos como definidores del estilo, como los arcos de herradura, las cornisas de modillones o las bóvedas gallonadas. Son elementos arquitectónicos comunes a estas iglesias y que no aparecen en otras de la misma época. También es significativa la articulación de los espacios interiores y la volumetría exterior, que suele ser muy característica y diferente de otros edificios coetáneos. Por ello, excluiremos de nuestro estudio una serie de iglesias que Gómez-Moreno cita como mozárabes y que siguiendo el criterio más habitual consideramos como prerrománicas, como las asturianas, que deben ser objeto de un estudio específico, por su singularidad.

La base de datos que manejaremos está formada por 54 iglesias mozárabes, aunque en el tratamiento estadístico solo vamos a considerar 49, que se aportan en la tabla 1. González-García y Belmonte aportan una base de datos de 57 iglesias mozárabes a las que añaden 15 iglesias del Serrablo, pero consideramos que algunas de ellas no tienen las características que hemos definido. Eliminamos del conjunto las iglesias de Santa María de Melque y las de San Pedro, Santa María y San Miguel de Tarrasa, puesto que, aunque la mayor parte de su edificación se suele considerar mozárabe, sus arranques son visigóticos y, por tanto, su orientación fue fijada de acuerdo con criterios anteriores. Tampoco San Román de Toledo, cuya fábrica actual es mudéjar, aunque conserva restos mozárabes y posiblemente su origen sea visigodo. En todo caso, no tenemos datos que permitan afirmar con seguridad que su trazado corresponde a época mozárabe $o$, si se prefiere, de repoblación. Por último, también excluimos de nuestro estudio algunas iglesias que son claramente prerrománicas, pero que la mayoría de los autores no consideran mozárabes, como las de Berbetouros, Corticela, Olleros, Santa Coloma de Andorra, Coruña del Conde y algunas otras. Consideramos que, pese a datarse en el siglo X, no presentan ninguna de las características formales mozárabes. Entendemos que las conclusiones que se puedan obtener serán más fiables en la medida en que se analice un conjunto de iglesias que sean consideradas como mozárabes por la mayoría de los estudiosos, con sus características arquitectónicas propias. Naturalmente este es un punto que siempre estará sujeto a discusión. De hecho, disponemos de los datos de orientación de las iglesias mencionadas y sin dificultades se podrían incorporar a la base de datos.

También se incorporan a la base de datos las iglesias conocidas con el nombre de iglesias del Serrablo, un notable conjunto construido en la cuenca alta del río Gállego en Huesca ${ }^{18}$. No hay unanimidad en la definición de estos templos, pues una tendencia los considera construidos por mozárabes y otra los considera prerrománicos o incluso del primer románico. Por nuestra parte, tanto por su época de construcción como

7 Bango Torviso "Arquitectura de la décima centuria".

18 Se incluyen también en la base de datos que aportan González-García y Belmonte, aunque no hacen un estudio estadístico diferenciado. 
por alguno de sus elementos definitorios, hemos considerado conveniente incluir estas iglesias en nuestra base de datos, si bien haremos un estudio específico y diferenciado de las mismas, precisamente por criterios de coherencia estadística.

En cambio, no se incluyen en la base de datos las iglesias construidas en el siglo $\mathrm{X}$ en la Septimania. Su adscripción a lo que se suele conocer como arquitectura mozárabe se basa en su datación en el siglo X, así como en el uso del arco de herradura. Sin embargo, en nuestra opinión, parecen más próximas estilísticamente a modelos visigodos que mozárabes. Además la Septimania fue una zona de fuerte influencia visigoda, hasta el punto de que su denominación habitual tras el reinado de Carlomagno fue Gothia. Estas cuestiones, unidas a la confusa situación política de la Septimania en el siglo $\mathrm{X}$, aconsejan un estudio específico de estas iglesias, que no se aborda en el presente trabajo.

\section{Medición de la orientación y factores que intervienen}

Como se ha señalado, una vez medida la orientación del edificio, un cálculo astronómico relativamente sencillo permite conocer con precisión el día en el que fue orientado. Nuestras mediciones corresponden a un amplio abanico, que cubre casi todo el año. El orto varía entre el punto $\mathrm{A}$, que corresponde al solsticio de invierno, y el punto $\mathrm{B}$, que corresponde al solsticio de verano, pasando dos veces por el punto vernal, en los dos equinoccios. Naturalmente cada punto del horizonte marca el orto solar en dos días del año, uno antes y otro después del equinoccio correspondiente. Las orientaciones entre los puntos E y B corresponden a la primavera y verano y las que están entre A y E corresponden al otoño y al invierno. Como se verá, las iglesias mozárabes tienen orientaciones que son mayoritariamente de primavera y verano. Es una característica de estas iglesias que también aparece en las asturianas y que no se produce en otras.

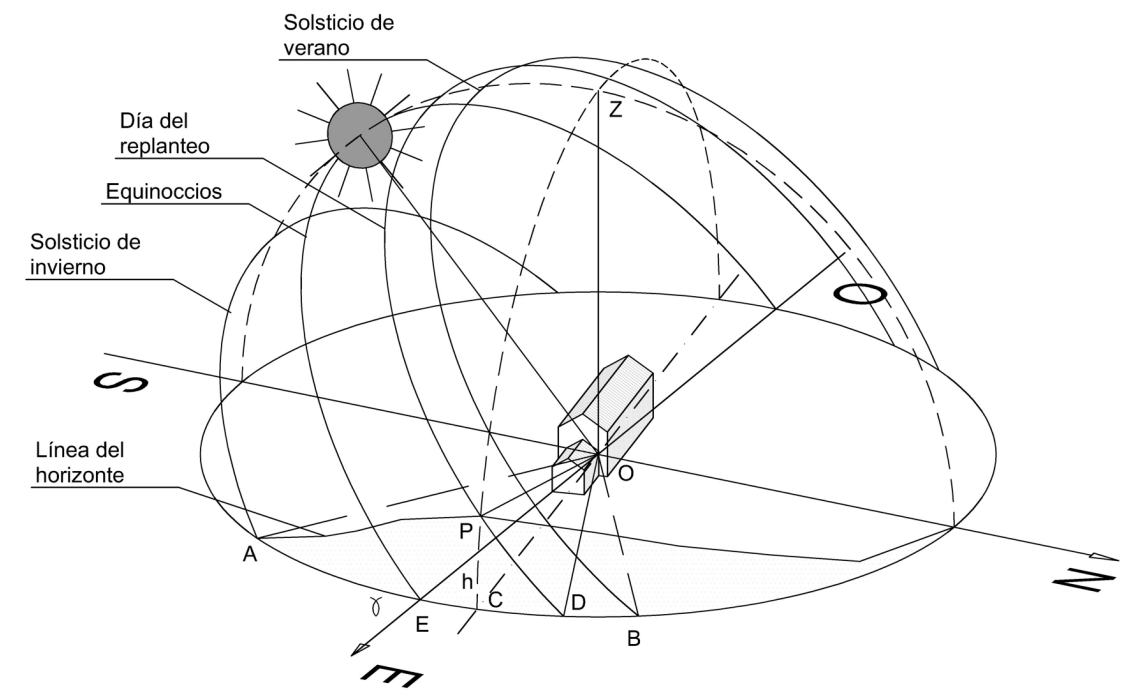

Figura 2. Trayectorias aparentes del Sol en el año. 
Existe un problema para determinar la orientación real: cuando el horizonte no es plano, la salida del sol se produce cuando ya ha recorrido una parte de su trayectoria. De la misma manera, el ocaso se produce cuando todavía falta parte de la trayectoria solar. Este efecto es especialmente importante en zonas montañosas o con obstáculos naturales próximos al edificio. En nuestras latitudes supone una corrección horizontal ligeramente menor que el ángulo de pendiente y afecta a casi todos los edificios estudiados de forma notable.

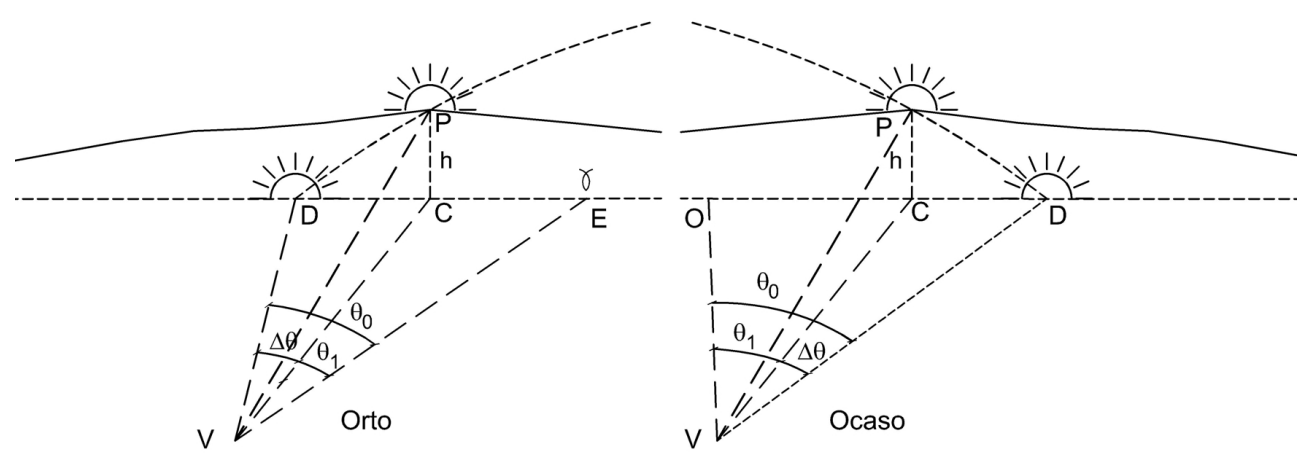

Figura 3. Posición del sol en un horizonte en pendiente al orto y al ocaso.

Si el horizonte tiene una cierta altura (h), la posición del orto (u ocaso) solar que podían observar los constructores de la iglesia se produce en el punto $\mathrm{P}$. La posición del templo se define por el punto $\mathrm{C}$, lo que marca la orientación medida, pero en realidad el día en el que se hizo el replanteo correspondería a una salida del Sol en un horizonte plano en el punto $\mathrm{D}$, que es lo que hemos definido como posición del orto (u ocaso) real.

Los datos necesarios son la orientación y la altura del horizonte, que afecta considerablemente la dirección del orto real, como se ha visto. La orientación se mide con brújula por el ángulo que forma con la dirección norte y la altura del horizonte se mide con clinómetro. También necesitamos conocer la latitud del lugar, puesto que las trayectorias solares dependen de ella. En el problema intervienen además factores menores, como son la excentricidad de la órbita aparente del Sol o los movimientos de precesión o nutación de la Tierra. De ellos solo tiene una influencia muy ligera la excentricidad de la órbita. El modelo de cálculo lo tiene en cuenta, pese a su poca incidencia. Otro factor que interviene en el problema es el efecto del radio solar y la refracción atmosférica. El radio solar es de 16' y hay que añadirle 34' debido a la refracción atmosférica. Esto supone que cuando se observa la salida del sol en realidad está 34' más bajo del horizonte. Esta diferencia no se ha tenido en cuenta, sino que se aplica al error general admitido, puesto que las posibles enfilaciones tendrían, sin duda, un error superior.

La variabilidad en las medidas de la orientación es una prueba de que el criterio de replanteo solo puede estar justificado por el orto solar. Lo razonable es pensar que el replanteo se hizo fijando la dirección de la salida del sol y probablemente se realizó con estacas o barras de hierro que se hincarían en el suelo definiendo la línea este-oeste. Es una técnica constructiva bien conocida desde la construcción egipcia y que está documentada ampliamente en la construcción griega y sobre todo en la ro- 
mana, que es el precedente inmediato ${ }^{19}$. Por otra parte, aparece en el álbum de Villard de Honnecourt, por lo que es razonable suponer una continuidad, dada su sencillez. A partir de esa línea se trazaban los ejes perpendiculares a ella y el trazado de dichas perpendiculares, que no es sencillo, definía la calidad de la ejecución. Podemos encontrar trazados sumamente irregulares, como San Juan de la Peña o el monasterio de Suso, pero en general la arquitectura mozárabe suele estar bien trazada y aparejada. En algunos casos, como en San Miguel de Celanova, la calidad constructiva es excepcional. La alineación este-oeste puede definirse por medio de la sombra de una sola estaca, pero el sistema anterior nos parece más probable. De lo que podemos estar seguros, es que la orientación se fijaba por el ábside. Siempre era lo primero en ser construido, puesto que permitía ya iniciar el culto. Así pues, la orientación de las iglesias se fijará siempre por el eje del ábside y es la alineación que debemos medir, pese a sus dificultades prácticas.

La toma de medidas de la orientación se ha realizado por los autores utilizando una brújula de precisión Recta DS-50. Los dispositivos basados en GPS han resultado poco adecuados al ser empleados en interiores, por lo que se abandonó su uso. Se han tomado las orientaciones de eje del ábside y, en su caso, de la nave de la iglesia en todos los casos en los que han sido accesibles. Cuando no es posible acceder al interior, se pueden tomar las orientaciones desde el exterior en varios puntos, de los que consideramos más significativos los laterales del ábside, como se indica en la figura 4, que corresponde al pequeño oratorio de San Miguel de Celanova, en Orense ${ }^{20}$. En este caso, la planta es bastante regular, lo que no es lo habitual. La brújula utilizada da una precisión de $0,5^{\circ}$. Con todo, las irregularidades del edificio y la falta de referencias fijas aconsejan estimar en los cálculos un posible error de $\pm 1^{\circ}$ con respecto al valor medido.
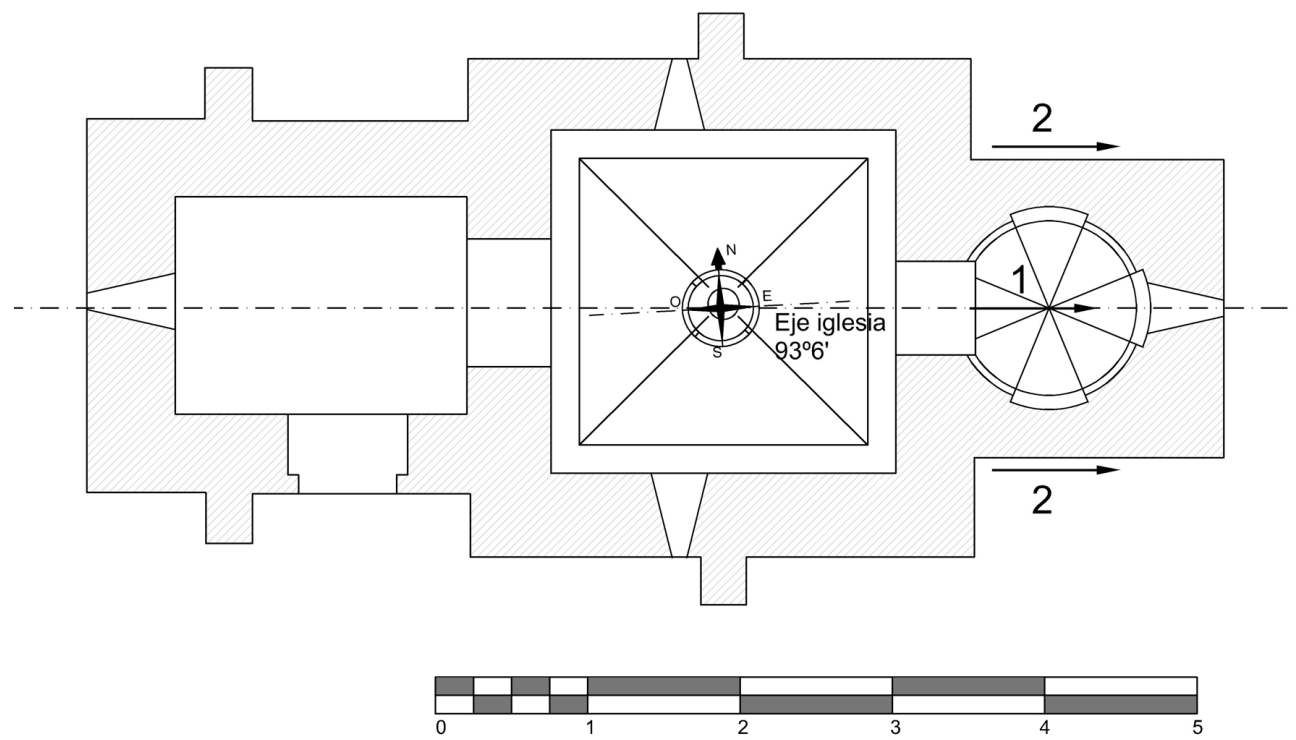

Figura 4. Puntos de toma de la orientación en la iglesia de San Miguel de Celanova.

19 Existen testimonios de la ceremonia egipcia del estiramiento de la cuerda, vinculada a la diosa Seshat, desde la I Dinastía, y también aparece en la obra de Vitrubio.

20 Esta orientación concreta se ha tomado con precisión desde el interior, pero, en otros casos, no es posible. 
Una brújula mide la posición del norte magnético, no la del norte geográfico, que es el que nos interesa para nuestros cálculos. Debe ser corregido con la declinación magnética, que es el ángulo formado por ambos nortes y que está disponible con diversas aplicaciones. La declinación magnética varía con las coordenadas geográficas (longitud y latitud) del lugar y la fecha de medición. Antes de 2011 se utilizó para el cálculo de la declinación el programa Geomag, que todavía utilizamos para iglesias situadas fuera de España ${ }^{21}$. En territorio nacional se utilizó a partir de 2011 el calculador del Instituto Geográfico Nacional, por ser más preciso. Cualquiera de ellos proporciona una precisión muy superior a lo necesario. El norte geográfico será el ángulo medido (referido al norte magnético) más la declinación magnética con su signo. No todas las medidas tomadas pueden ser validadas. Existen lugares con anomalías magnéticas naturales que pueden alterar las mediciones de la brújula. También existen elementos de hierro capaces de alterar la medición. Es necesario estar seguro de que las medidas tomadas son fiables. En este caso, se han contrastado las medidas con las fotos aéreas, disponibles en la aplicación Iberpix del Instituto Geográfico Nacional ${ }^{22}$. Con todo, es un recurso que debe ser empleado con prudencia, dados los posibles errores que presenta. Solo se emplea cuando se detectan anomalías magnéticas, que no se han observado en estas iglesias en concreto, aunque sí en otras.

Como hemos señalado, es esencial medir la pendiente del horizonte. Hemos utilizado un medidor láser de la marca Leica, modelo DISTOTM D8 con clinómetro digital con una precisión de $0,05^{\circ}$. Pero esta medida no siempre es posible, pues pueden existir edificaciones u obstáculos posteriores que la impiden. En estos casos, se ha recurrido al visor Iberpix, ya mencionado, que proporciona la distancia al elemento que define el horizonte y la diferencia de nivel entre dicho elemento y la iglesia. Con ello se obtiene la tangente del ángulo vertical que permite corregir la posición real del orto solar.

Con los datos de la latitud del lugar, la orientación del templo y la altura del horizonte, es posible calcular con gran precisión el día en el que templo fue replanteado usando el programa de ordenador ORIENT 2.0. Los cálculos se hacen en base al calendario juliano, que era el que utilizaban los constructores y comitentes en su época. Lo que cabe preguntarse es si ese día tenía algún significado especial que responda a alguno de los posibles criterios que se han indicado: simbólico, topográfico, litúrgico o constructivo.

\section{Orientación de las iglesias mozárabes}

Se han medido las orientaciones de 53 iglesias mozárabes en España y una en Portugal (San Pedro de Lourosa). La mayoría se corresponde con posiciones posibles del orto solar, aunque hay algunas singularidades muy notables. Generalmente se conoce la advocación de las iglesias mozárabes, salvo la basílica de Bobastro (Málaga), que además es la única construida en territorio musulmán, junto con la iglesia de

21 GeoMag (Geographic Magnetic Calculator) es un programa de uso libre desarrollado por Garry Petrie de Nestucca Drive, Portland, Oregón de gran utilidad e interés.

22 http://www.ign.es/iberpix2/visor/. Es una excelente aplicación de gran utilidad, que permite obtener fotos aéreas, mapas topográficos y medir distancias y pendientes de cualquier punto de España. 
Bobastro descubierta recientemente y la ermita de la Virgen de la Cabeza en Ronda, ya citadas. En las restantes y, al contrario de otros casos que hemos estudiado, no hemos encontrado iglesias cuya advocación no coincida con la actual. Sin embargo, hay un caso que suscita alguna duda. La festividad de Santiago, según el calendario mozárabe, es el 30 de diciembre, aunque también existe la festividad del martirio de Santiago el 25 de julio. Solo afecta a una iglesia, Santiago de Peñalba, pero de tal importancia que entendemos que se deben estudiar ambas fechas posibles ${ }^{23}$.

\subsection{Análisis de los criterios de orientación de las iglesias mozárabes}

En primer lugar haremos una estadística de los valores medidos de la orientación real, corregida con el efecto de la pendiente del horizonte, formando dos series estadísticas. La serie 1 corresponde a las iglesias mozárabes con exclusión de las del Serrablo, que se estudiarán aparte. La serie 2 corresponde a la totalidad de iglesias medidas, incluyendo las del Serrablo. Los resultados los podemos resumir en la siguiente tabla.

Tabla 1. Estadísticas de las orientaciones de las iglesias mozárabes.

\begin{tabular}{|c|c|c|c|c|c|c|c|c|c|c|c|}
\hline & \multicolumn{11}{|c|}{ Número de iglesias orientadas según el ángulo } \\
\hline Orientación en ${ }^{\circ}$ & $<63$ & $\begin{array}{l}63 \text { a } \\
69\end{array}$ & $\begin{array}{l}69 a \\
75\end{array}$ & $\begin{array}{l}75 \mathrm{a} \\
81\end{array}$ & $\begin{array}{l}81 \text { a } \\
87\end{array}$ & $\begin{array}{l}87 \text { a } \\
93\end{array}$ & $\begin{array}{l}93 \text { a } \\
99\end{array}$ & $\begin{array}{l}99 \mathrm{a} \\
105\end{array}$ & $\begin{array}{l}105 \mathrm{a} \\
111\end{array}$ & $\begin{array}{l}111 \mathrm{a} \\
117\end{array}$ & $>117$ \\
\hline $\mathrm{n}^{\circ}$ serie 1 & 9 & 4 & 1 & 3 & 8 & 1 & 5 & 3 & 0 & 2 & 1 \\
\hline $\mathrm{n}^{\mathrm{o}}$ serie 2 & 9 & 5 & 2 & 5 & 12 & 1 & 8 & 3 & 0 & 3 & 1 \\
\hline Frec.\% 1 & 24,3 & 10,8 & 2,7 & 8,1 & 21,6 & 2,7 & 13,5 & 8,1 & 0,00 & 5,4 & 2,7 \\
\hline Frec.\% 2 & 18,4 & 10,2 & 4,1 & 10,2 & 24,5 & 2,0 & 16,3 & 6,1 & 0,00 & 6,1 & 2,0 \\
\hline
\end{tabular}

De esta estadística excluimos los dos datos más atípicos, que son los que corresponden a las iglesias de Hérmedes de Cerrato y a Valdueza, con lo que obtenemos un conjunto razonablemente homogéneo. En la tabla 2 se aporta el valor medio de las orientaciones reales, la desviación típica y el día del año juliano, al que corresponde en fechas del siglo X. Se aportan los datos de las iglesias mozárabes, excluyendo las del Serrablo, la estadística del Serrablo y la estadística total. Se observa una clara tendencia a usar orientaciones que corresponden a la primavera y el verano. Las orientaciones de invierno son una clara minoría. En el caso de las iglesias del Serrablo se observa una tendencia a la orientación en fechas algo más tempranas.

23 En el Códice Calixtino se menciona una festividad de Santiago el 25 de marzo, que no aparece en los calendarios mozárabes y visigodos consultados. Al ser el Calixtino muy posterior, se estima razonable limitarse a las fechas citadas. 
Tabla 2. Promedio y desviación típica de las orientaciones reales.

\begin{tabular}{|l|c|c|c|}
\hline & Promedio & Desv. típica & Día \\
\hline Iglesias mozárabes & $80,17^{\circ}$ & $19,91^{\circ}$ & 4 abril \\
\hline Iglesias Serrablo & $87,21^{\circ}$ & $12,74^{\circ}$ & 21 marzo \\
\hline Total & $81,82^{\circ}$ & $18,88^{\circ}$ & 1 abril \\
\hline
\end{tabular}

Esta distribución es bastante similar a la observada en las iglesias prerrománicas, si bien en ellas hay una mayor frecuencia de las orientaciones menores de $60^{\circ}$, llegando incluso a orientaciones que no corresponden al orto solar. En las iglesias mozárabes este caso aparece con claridad en San Millán de Suso y San Baudelio de Berlanga, y dentro de un margen de error que podría atribuirse al replanteo en la Asunción de Villarmún y San Adrián de Boñar. En todas estas iglesias, haremos un estudio específico para ver si pueden estar determinadas, no por el orto solar, sino por el ocaso. La media de los valores de orientación de las iglesias mozárabes supone un ángulo mayor al de otras iglesias prerrománicas, cuyo valor medio es algo inferior a $70^{\circ}$. Esto supone una tendencia a orientarlas en épocas más tardías del año que las mozárabes. En cuanto a las iglesias románicas, se observan medias más próximas a $90^{\circ}$, es decir, que en general se orientaban en fechas más tempranas. La desviación estándar de las iglesias mozárabes es alta, pero menor que en otros casos estudiados. Entendemos que este análisis estadístico es razonablemente fiable, pero, pese a ello, debe ser contemplado más como tendencia que como un dato preciso. Todos los datos que se aportan han sido medidos in situ por los autores y se incluyen en una base de datos propia de 977 iglesias paleocristianas, visigodas, asturianas, mozárabes, prerrománicas y románicas. Cuando se hagan razonamientos estadísticos comparativos, nos estaremos refiriendo a esta base de datos general.

En primer lugar, vamos a definir la base de datos utilizada para el estudio específico de las iglesias mozárabes. Se aportan la totalidad de las iglesias medidas, a excepción de las del Serrablo, que se estudian aparte, y se señalan en cursiva aquellas que han sido excluidas de los análisis estadísticos por las razones expuestas. En la base de datos se incluyen dos veces San Clemente de la Valdueza, considerando las dos posibles orientaciones al este o al oeste, y Santiago de Peñalba, en la que se analizan las dos posibles festividades indicadas. Estos casos se señalan también en cursiva para evitar confusiones. Para poder visualizar la situación geográfica de las distintas iglesias, se ordenan por su longitud geográfica, de oeste a este. En la tabla se indica la orientación real de la iglesia, considerando la medida in situ con la corrección de la declinación magnética, la posición del orto real considerando el efecto de la pendiente del terreno, el orto simbólico, es decir, la posición del orto solar el día de la festividad del santo titular, la diferencia entre ambas posiciones que nos indica la desviación de la posible orientación simbólica y la pendiente medida.

Tabla 3. Orientaciones de las iglesias mozárabes.

\begin{tabular}{|l|l|r|r|r|r|r|}
\hline Iglesia & Lugar & Orient. real & Orto real & Orto simb. & Diferencia & Pend. $^{\text {o }}$ \\
\hline S. Antolín & Toques. & 78.5 & 70.5 & 81.3 & -10.8 & 8.0 \\
\hline S. Miguel & Celanova. & 93.5 & 93 & 95.7 & -2.7 & 0.44 \\
\hline
\end{tabular}




\begin{tabular}{|c|c|c|c|c|c|c|}
\hline S. Pedro & Lourosa & 82.5 & 81 & 57.7 & 23.3 & 1.65 \\
\hline S. Martín & Pazó & 99.5 & 96 & 116 & -20 & 3.75 \\
\hline Sta. Eufemia & Ambía & 89 & 86 & 88.7 & -2.7 & 3.55 \\
\hline S. Pedro & Rocas (Oeste) ${ }^{24}$ & 271.5 & 271.5 & 302.3 & -30.8 & 0 \\
\hline Sta. María & Mixós & 65 & 61 & 72.5 & -11.5 & 3.65 \\
\hline Santo Tomás & Ollas. & 89.5 & 86 & 123 & -37 & 4.05 \\
\hline S. Clemente & Valdueza (este) & 94.5 & 61.5 & 117.4 & -55.9 & 36.4 \\
\hline S. Clemente & Valdueza (oeste) & 274.5 & 294.5 & 297.4 & -2.9 & 21.75 \\
\hline Santiago & Peñalba $\mathrm{a}^{25}$ & 83.5 & 65.5 & 112.2 & -46.7 & 19.45 \\
\hline Santiago & Peñalba ${ }^{26}$ & 83.5 & 65.5 & 63.5 & 2 & 19.45 \\
\hline S. Salvador & Destriana & 68 & 68 & 119.8 & -51.8 & 0 \\
\hline La Asunción & Camarzana de Tera & 111.5 & 111.5 & 72.5 & 39 & 0 \\
\hline S. Salvador & Palat del Rey & 63.5 & 62 & 119.8 & -57.8 & 1.45 \\
\hline La Asunción & Villarmún & 51.5 & 50.5 & 72.5 & -22 & 0.8 \\
\hline S. Adrián & Boñar & 58 & 51.5 & 56.9 & -5.4 & 5.85 \\
\hline S. Miguel & Escalada & 99.5 & 98.5 & 95.7 & 2.8 & 0.7 \\
\hline S. Pelayo & Bodas & 118.5 & 116.5 & 57.3 & 59.2 & 2.1 \\
\hline S. Cebrián & Mazote & 92 & 87 & 117 & -30 & 5.4 \\
\hline Sta. María & Wamba & 68.5 & 65.5 & 72.5 & -7 & 3.15 \\
\hline S. Fructuoso & Valoria del Alcor & 60.5 & 60 & 116 & -56 & 0.9 \\
\hline Desconocido & Bobastro & 73.5 & 59 & & 59 & 18.0 \\
\hline Sta. María & Lebeña & 85.5 & 67 & 72.5 & -5.5 & 19.45 \\
\hline S. Román & Toledo & 77 & 77 & 98.7 & -21.7 & 0 \\
\hline Sta. María & Melque & 61 & 60.5 & 72.5 & -12 & 0.5 \\
\hline Sta. María & S. Cristóbal de Boedo & 111.5 & 100.5 & 72.5 & 28 & 11.0 \\
\hline S. Román & Moroso & 93 & 84.5 & 98.7 & -14.2 & 8.9 \\
\hline Sta.Leocadia & Helguera & 90.5 & 83 & 122.8 & -39.8 & 8.1 \\
\hline Sta. María & Hermedes de Cerrato & 337 & 337 & 72.5 & 264.5 & 0 \\
\hline Sta. María & Retortillo & 105 & 104 & 72.5 & 31.5 & 1.0 \\
\hline Sta. Centola & Siero & 90 & 86 & 66.9 & 19.1 & 3.95 \\
\hline Sta. Marina & Rodilla & 65.5 & 58 & 61.7 & -3.7 & 7.6 \\
\hline Sta. Cecilia. & Santibánez de Val & 85.5 & 81.5 & 119.5 & -38 & 4.3 \\
\hline S. Pelayo & Arlanza & 97.5 & 96 & 57.3 & 38.7 & 1.95 \\
\hline S. Félix & Oca & 83.5 & 77 & 69.8 & 7.2 & 7.0 \\
\hline S. Millán & Suso & 55.5 & 40 & 116.3 & -76.3 & 14.15 \\
\hline S. Baudelio & Berlanga. & 51.5 & 50 & 60.8 & -10.8 & 1.3 \\
\hline S. Juan Bta. & Peña ${ }^{27}$ & 303.5 & 313 & 236.5 & 76.5 & 8.6 \\
\hline S. Miguel & Olérdola & 93.5 & 93.5 & 95.7 & -2.2 & 0 \\
\hline
\end{tabular}




\begin{tabular}{|l|l|r|r|r|r|r|}
\hline Sta. María & Matadars & 89 & 77.5 & 72.5 & 5 & 12.75 \\
\hline S. Quirce & Pedret & 109.5 & 105 & 56.9 & 48.1 & 4.8 \\
\hline S. Miguel & Tarrasa & 80 & 80 & 95.7 & -15.7 & 0 \\
\hline S. Pedro & Tarrasa & 81 & 81 & 57.7 & 23.3 & 0 \\
\hline Sta. María & Tarrasa & 80 & 80 & 72.5 & 7.5 & 0 \\
\hline S. Julián & Buada & 84.5 & 84.5 & 99.9 & -15.4 & 0 \\
\hline
\end{tabular}

El primer criterio del que vamos a analizar su posible validez es el criterio simbólico, que supone que las iglesias se orientaron en la festividad de su patrón. Para ello se utiliza una carta solar anual, en la que se indican las posiciones del orto solar a lo largo del año durante el siglo X, que corresponde al período de construcción de la mayor parte de los edificios estudiados. Las fechas están definidas según el calendario juliano. Se han fijado las posiciones de la curva de ortos a $39^{\circ} \mathrm{N}$ que es una latitud intermedia en la zona estudiada. No le quita generalidad al estudio, puesto que la influencia de la latitud es reducida a este efecto ${ }^{28}$. Sobre esa carta se sitúa la posición de cada iglesia, incluyendo, en este caso, las iglesias del Serrablo, fijando como día del año el de la fiesta de su patrón y la dirección en la que está orientada. De haber sido replanteada en ese día concreto su posición en el gráfico debiera coincidir, siquiera

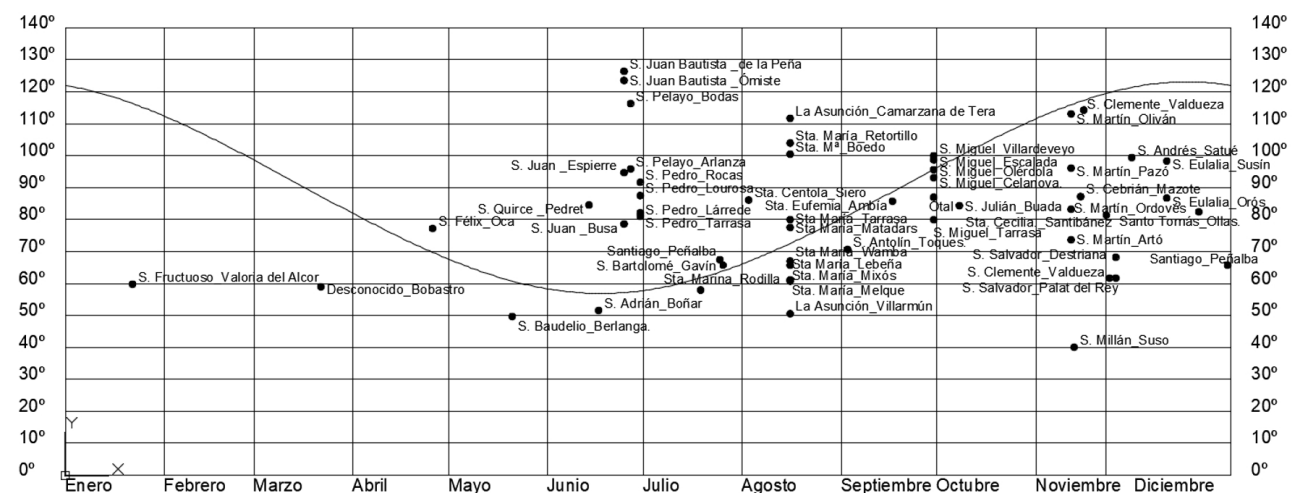

aproximadamente con la curva de ortos.

Figura 5. Correlación entre las posiciones de las iglesias mozárabes y el orto solar.

Ese día debe definirse de acuerdo con el calendario vigente en la época de construcción de la iglesia y eso supone algunos problemas. Se han tomado como referen-

24 La orientación está fijada por el oeste, al ser una iglesia rupestre. La orientación medida hacia el este es de 91,7º, que es la que se toma a efectos de estadística.

25 Corresponde a la festividad del 30 de diciembre.

26 Corresponde a la festividad del 25 de julio.

27 La orientación medida hacia el este es de $123,5^{\circ}$, que es la que se considera a efectos estadísticos, pero, puesto que necesariamente se orientó al ocaso, se indica la orientación al oeste en ese mismo día.

28 Puesto que se admitirá para el criterio simbólico un margen de diferencia muy amplio, la influencia de la latitud, en este caso, tiene una incidencia muy escasa y se ha aplicado solo al gráfico. Los cálculos que se aportan sí tienen en cuenta el efecto de la latitud real de los edificios. 
cias las obras de Agustí y Voltes y Vives Castell en la que se definen las festividades de los distintos calendarios medievales españoles ${ }^{29}$. En la obra de Agustí y Voltes, se incluye el calendario hispano-mozárabe y una recopilación de los calendarios medievales españoles. La obra de Vives se refiere específicamente al calendario visigodo, que, sin duda, era conocido y que tiene grandes similitudes con el calendario mozárabe, que es nuestra referencia principal.

La simple observación de la figura muestra con claridad que no hay una correlación clara, si bien los resultados son mucho mejores que en otros estilos arquitectónicos. Si fijamos una variación de $\pm 10^{\circ}$, sin duda notable, aparecen 17 iglesias en esa franja, es decir, un 31\% aproximadamente. Es un porcentaje reducido, pero aun así claramente superior a otros casos, como las visigóticas o las románicas. Incluso fijando una margen de $\pm 5^{\circ}$, que ya puede considerarse dentro de los errores posibles de replanteo, encontramos 10 iglesias, es decir un 18,5\%, lo que constituye un resultado realmente notable, en comparación con otros casos. Tampoco se ha encontrado ninguna coincidencia especial en las fechas del 1 de abril o 25 de julio, ya comentadas.

En las iglesias mozárabes se observa una mayor dispersión de las advocaciones que se dedican a un mayor número de santos diferentes que en otros períodos. Las más numerosas son Santa María (15 de agosto) con 11, San Miguel (29 de septiembre) con 6, San Juan Bautista (24 junio) con 4, San Pedro (29 de Junio) con 4 y San Martín (11 de noviembre) con 3. Se señalan específicamente estas festividades tanto por su mayor frecuencia como porque coinciden en todos los calendarios medievales consultados y, en especial, en el calendario mozárabe. También llama la atención que la mayoría de las advocaciones corresponden a festividades de la segunda mitad del año.

Siempre existe una duda en la advocación a santa María, puesto que en los calendarios medievales figuran cuatro fiestas principales de la Virgen. Son la Anunciación (25 marzo), la Purificación (2 febrero), la Asunción (15 agosto) y la Natividad (8 septiembre). Lógicamente existe siempre la duda de si en realidad la iglesia tenía alguna de esas advocaciones concretas que se ha perdido con el tiempo. Sin embargo y extrapolando los datos de las iglesias románicas, solo hemos encontrado cuatro dedicadas a la Natividad y una a la Purificación, mientras que la Asunción presenta 24 ejemplos, con esa advocación concreta. En el caso de las iglesias mozárabes, hay 9 dedicadas a santa María, sin una advocación especial, y 2 dedicadas específicamente a la Asunción. Por ello, hemos considerado en todos los casos la fiesta el 15 de agosto, aun reconociendo la incertidumbre de esta opción.

Un caso especialmente interesante es el de la basílica de Bobastro en Las Mesas de Villaverde (Málaga), de la que no conocemos su advocación, puesto que la destrucción de todos los restos a causa de la rebelión de Omar Ben Hafsún fue completa y sistemática. Su orientación es de $73,5^{\circ}$ y la pendiente del horizonte es de $18^{\circ}$, lo que supone que la posición del orto real es $59^{\circ}$. Sorprende este hecho porque en muchas de las referencias consultadas se cita sistemáticamente que está orientada al este exacto, cuando la diferencia es de $16,5^{\circ}$, claramente apreciable ${ }^{30}$. La fecha en la que pudo haber sido orientada es prácticamente el solsticio de verano. A falta de mejores hipótesis, podríamos suponer que se orientó en la festividad de San Juan Bautista,

29 Agustí y Casanovas y Voltes, Manual de cronología española y universal; Vives Castell, Santoral visigodo en calendarios e inscripciones, pp. 31-58.

30 González-García y Belmonte, "The Orientation of Pre-Romanesque Churches in the Iberian Peninsula" proporcionan unas medidas realistas. 
lo que hubiera sido lógico, al tratarse de un profeta muy respetado por ambas religiones, aspecto de especial interés si tenemos en cuenta las dificultades que tuvo Omar Ben Hafsún entre sus partidarios por su cambio de religión. Es una hipótesis atractiva, pero no tenemos ninguna prueba.

El segundo criterio, que hemos llamado topográfico, consiste en suponer que las iglesias se replantearon de acuerdo con los condicionantes simbólicos, geográficos o paisajísticos del lugar. No hemos encontrado una correlación que podamos entender como sistemática, pero sí algunas coincidencias interesantes. La orientación hacia un pico montañoso se produce muy claramente en la iglesia rupestre de San Pedro de Rocas (Ourense), situada en una cueva abierta al oeste hacia un pico de A Coutada, pero aquí los condicionantes los define la propia cueva. Son muy notables las orientaciones hacia picos montañosos en las iglesias del Serrablo, que ya se comentarán. También las iglesias de Berlanga y Suso tienen orientaciones que pueden explicarse por los condicionantes simbólicos del lugar. En todo caso, son situaciones atípicas, que no permiten justificar un uso generalizado de este criterio.

El tercer criterio, que hemos llamado litúrgico, consiste en suponer que las iglesias se replantearon de forma preferente durante la Pascua. Ya desde el Concilio de Nicea quedó establecido que la Pascua se celebraría el domingo de la primera luna llena de primavera, es decir, posterior al 21 de marzo. Así, el día más temprano para la Pascua sería el 22 de marzo, y el más tardío el 25 de abril, pero en fechas julianas, que en ese siglo tenían algo más de cinco días de retraso. Las orientaciones que corresponden al período pascual en fechas julianas en el siglo $\mathrm{X}$ son: Latitud $42^{\circ}$ entre $86^{\circ} 45^{\prime}$ ( 22 de marzo) y $69^{\circ} 46^{\prime}$ ( 25 abril).

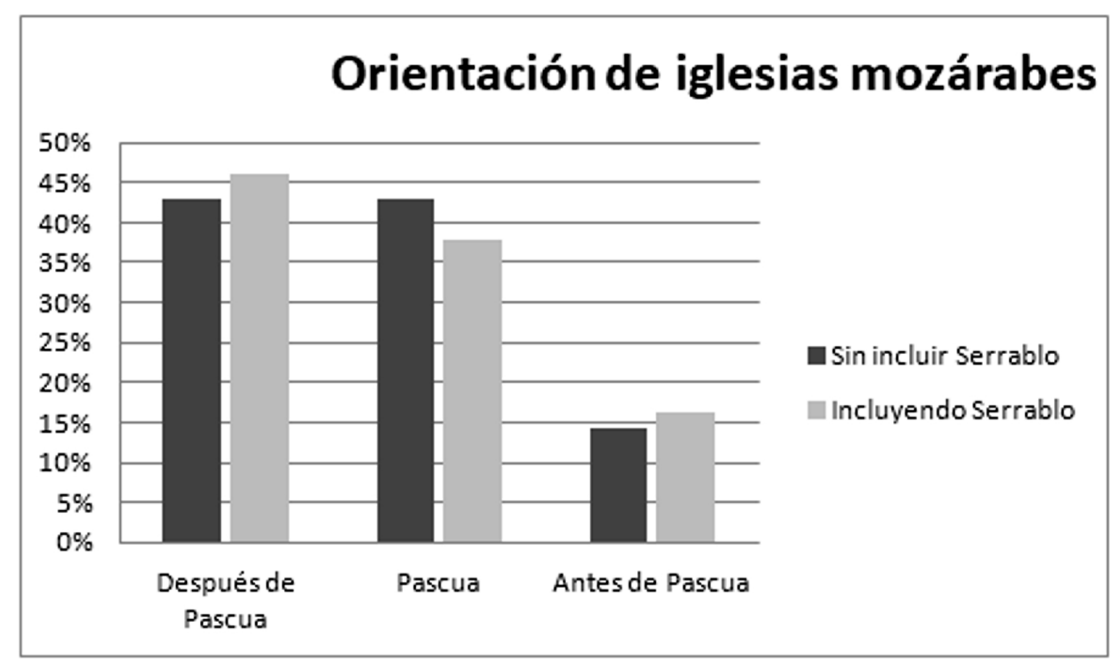

Figura 6. Análisis del criterio litúrgico para la orientación de las iglesias mozárabes.

En el caso de las iglesias mozárabes, el $45,95 \%$ corresponde a fechas posteriores a la Pascua, el 37,84\% corresponde a fechas comprendidas en el período pascual y el $16,22 \%$ a fechas anteriores a la Pascua. Si incluimos las iglesias del Serablo, 
el $42,86 \%$ son anteriores y el mismo porcentaje corresponde a la Pascua, siendo el $14,29 \%$ de fechas anteriores. Hay una diferencia notable con iglesias prerrománicas y románicas en las que hay una frecuencia claramente mayor de orientaciones antes de la Pascua. No hemos encontrado ninguna razón lógica que explique esta diferencia, bastante apreciable.

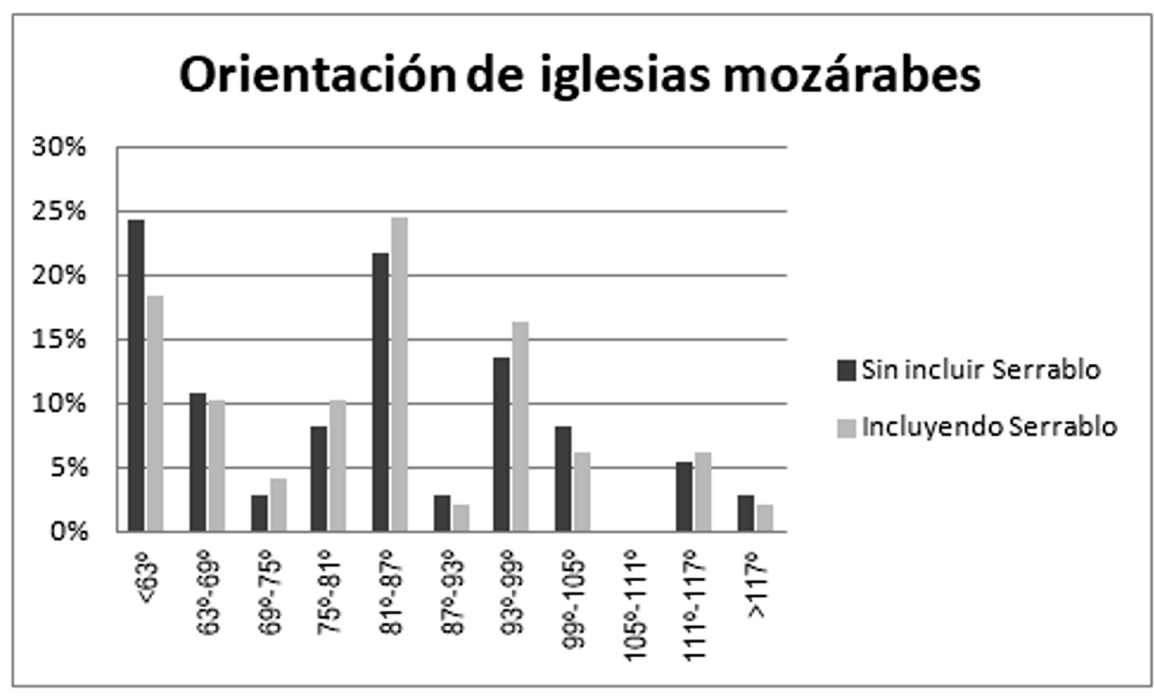

Figura 7. Estadística de las orientaciones de las iglesias mozárabes.

Por exclusión, todo apunta a que el criterio más probable es el constructivo, es decir, suponer que la iglesia se orientó el día del replanteo, con independencia de otras consideraciones simbólicas o litúrgicas. Es un criterio mayoritario, con la única oposición de Hinton, que, sin rechazarlo, hace una seria crítica al mismo ${ }^{31}$. Su razonamiento se basa en la existencia de orientaciones de invierno, cuando la experiencia indica que no debe iniciarse una obra de fábrica en esa época. De hecho, existen contratos de obra en épocas posteriores, que exigen iniciarla después de una determinada fecha, normalmente de primavera. El motivo es claro: es necesario que la temperatura sea suficiente para que el mortero pueda fraguar en buenas condiciones. Es un razonamiento correcto, pero no parece adecuarse a la construcción medieval, en la que eran frecuentes las pausas, por limitaciones de recursos económicos, falta de suministros, etc. Hubiera sido muy razonable realizar el replanteo en una fecha anterior para tener margen para realizar las excavaciones, que era una de las labores más duras en la construcción medieval. No hay ninguna razón constructiva que obligue a iniciar la obra inmediatamente después de su replanteo, para el que solo es preciso un día despejado, que permita determinar la posición del orto solar y sin lluvia para colocar estacas y cuerdas.

En el caso concreto de las iglesias mozárabes, debemos considerar que, fijando una fecha razonable a partir del 1 de marzo, el orto solar se produce a unos $99^{\circ}$ en las

31 Hinton, Church Alignment and Patronal Saint's Days, p. 217. 
latitudes estudiadas. Según nuestra estadística, esto supone que 42 iglesias, es decir, el $85,7 \%$ del total tienen orientaciones posteriores a esa fecha. Por el contrario, las siete iglesias con orientaciones claramente de invierno suponen solo el 14,3 ${ }^{\circ}$. Excluyendo Hérmedes de Cerrato, por lo atípico de su orientación, tenemos solo Santa María de San Cristóbal de Boedo, Santa María de Retortillo, la Asunción de Camarzana de Tera, San Martín de Oliván, San Pelayo de Bodas y San Juan Bautista de la Peña. Están todas ellas situadas en zonas con inviernos rigurosos, poco propicios para iniciar una obra. En el caso de San Juan de la Peña, es evidente que no pudo ser orientada al este, empotrado en la roca, sino que necesariamente tuvo que orientarse al oeste.

Son posibles dos hipótesis que puedan explicar estas orientaciones de invierno tan claramente inadecuadas desde el punto de vista constructivo. La primera de ellas es que, como hemos indicado, no tiene por qué coincidir el replanteo de la obra con la fecha de inicio. Es perfectamente factible que se fije la orientación un día concreto, que evidentemente ha de ser despejado y seco y, en ese día, se haga el trazado y encordado de la planta. A partir de esa fecha son necesarios trabajos de desbroce, excavación y acopio de materiales que pueden retrasar la obra hasta fechas más adecuadas. Pero también es posible otra explicación. Una orientación al este superior a $99^{\circ}$ indica que la iglesia fue orientada al orto solar en invierno, pero, en cambio, si esa misma orientación se hace al ocaso, nos indica una fecha de verano. Aunque, salvo en el caso de San Juan de la Peña, no hay ninguna prueba de que se hayan orientado al oeste, es perfectamente posible, lo que sería prueba de que el replanteo se hizo en verano, mucho más lógico en estas zonas.

\subsection{Las iglesias del Serrablo}

En la cuenca alta del río Gállego se construyeron entre los siglos X y XI un conjunto de iglesias con unas características arquitectónicas muy definidas y diferentes de las de otras zonas. En general, son iglesias pequeñas, de una sola nave, ábside semicircular, en muchos casos rematado por un baquetón de lajas colocadas verticalmente, que ocupan el espesor del muro, lo que constituye su detalle más característico. También aparecen elementos que suelen definir los edificios mozárabes, como los arcos de herradura o los huecos enmarcados por alfiz. Siempre han sido objeto de polémica, puesto que no hay unanimidad en considerarlas como tal. Es difícil solventar esa duda, en especial por la especial situación del reino de Aragón en esa época, muy amenazado por la taifa de Zaragoza, pero entendemos que, desde el punto de vista arquitectónico, su filiación mozárabe es muy probable y, por ello, los incluimos en este artículo. Con todo, su estudio se separa de otras iglesias mozárabes.

Nuestra base de datos incluye las principales iglesias del Serrablo, excluyendo dos de ellas que fueron trasladadas (Basarán y Santa María de Gavín) -y que, por tanto, desconocemos su orientación original- $\mathrm{y}$ alguna de ellas que ha resultado inaccesible, como San Pelay de Gavín. Pese a ello, consideramos que es una base lo suficientemente representativa como para permitir algunas conclusiones. 
Tabla 4. Orientaciones de las iglesias del Serrablo.

\begin{tabular}{|l|l|c|c|r|l|}
\hline Iglesia & Lugar & Orient. real & Orto real $^{\text {Pend. }^{\circ}}$ & Observaciones \\
\hline S. Bartolomé & Gavín & 87,5 & 67,5 & 21,95 & Hacia el pico Cuechas \\
\hline S. Martín & Artó & 75,5 & 73,5 & 1,93 & Hacia el pico de Tozal de las Canas \\
\hline S. Juan Bta. & $\begin{array}{l}\text { Omiste } \\
\text { (Rasal) }\end{array}$ & 830 & 76,5 & 7,45 & Hacia un pico montañoso: Sierra de Javierre \\
\hline S. Juan & Busa & 99,5 & 78,5 & 22,55 & Casi al pico de Peña Blanca \\
\hline S. Pedro & Lárrede & 99 & 82 & 18,20 & Próx. al pico de S. Quílez \\
\hline S. Martín & Ordovés & 86,5 & 83 & 4,50 & Ladera \\
\hline Sta. Eulalia & Orós Bajo & 101,5 & 86,5 & 16,05 & Hacia el pico Los Forcones \\
\hline S. Miguel & Otal & 101 & 87 & 15,50 & Entre dos picos de altura similar \\
\hline S. Juan & Espierre & 107,5 & 94,5 & 13,60 & Hacia el pico de Erata \\
\hline S. Andrés & Satué & 117 & 97,5 & 18,55 & Hacia el pico Corona de Satué \\
\hline Sta. Eulalia & Susín & 103,5 & 98,5 & 5,40 & Hacia un pico montañoso \\
\hline S. Martín & Oliván & 126 & 113 & 11,00 & Ladera \\
\hline
\end{tabular}

El análisis de este conjunto de iglesias, si bien la muestra es relativamente escasa, nos permite hacer algunas consideraciones de interés. En primer lugar, se observa que la mayoría de las iglesias están orientadas hacia un pico montañoso, en concreto siete, lo que supone el 60\% de las medidas. Dos de ellas, Busa y Lárrede, no apuntan exactamente el pico, pero lo hacen cerca, Otal apunta a una cresta montañosa entre dos picos, y solo Ordovés y Oliván lo hacen a una ladera. Hemos encontrado otras orientaciones hacia picos montañosos en varios ejemplos de iglesias altomedievales, pero nunca con tal frecuencia. Este interés en buscar la enfilación a un pico montañoso es citado por Sassin Allen como justificación del criterio topográfico en su ámbito de estudio, que son las iglesias románicas de Gales. No existe prueba alguna de una intencionalidad y, por otra parte, todas las orientaciones medidas son canónicas y el entorno es sin duda propicio. Con todo, es una notable coincidencia que es oportuno reseñar. En las iglesias del Serrablo todas las orientaciones medidas corresponden a posiciones posibles del orto solar. Es una singularidad, puesto que en otras iglesias mozárabes se producen orientaciones atípicas, como se verá a continuación. También en iglesias paleocristianas, visigodas, prerrománicas o románicas. El único grupo en el que no se han detectado, es precisamente en este. Por otra parte, se observa que la dispersión de orientaciones es notable y, dadas las fuertes pendientes, el orto real está claramente desviado hacia el norte con respecto a la orientación medida.

\subsection{Orientaciones atípicas}

De la base de datos que se aporta solo hay cuatro iglesias cuya orientación no corresponde con una posición posible del orto solar, que son las iglesias de San Millán de Suso (La Rioja), San Baudelio de Berlanga (Soria), la Asunción de Villarmún y San Adrián de Boñar, ambas en León. A ellas hemos de añadir el caso singular de la ermita de Santa María de Hérmedes de Cerrato (Palencia) y un caso que suscita muchas dudas, como es el de San Clemente de Valdueza (León). Las iglesias de Suso, Berlanga, Villarmún y Boñar tienen orientaciones incompatibles con la posición del orto solar, pero que pueden corresponder a posiciones del ocaso. Para ello, las vamos 
a situar sobre una carta solar que tomaremos con una latitud media de $42^{\circ}$, que es una referencia media para las iglesias señaladas y que no supone un error apreciable ${ }^{32}$. Para determinar las posiciones reales del orto y el ocaso consideramos la pendiente en ambas direcciones.

$42,0^{\circ} \mathrm{N}$ Latitud

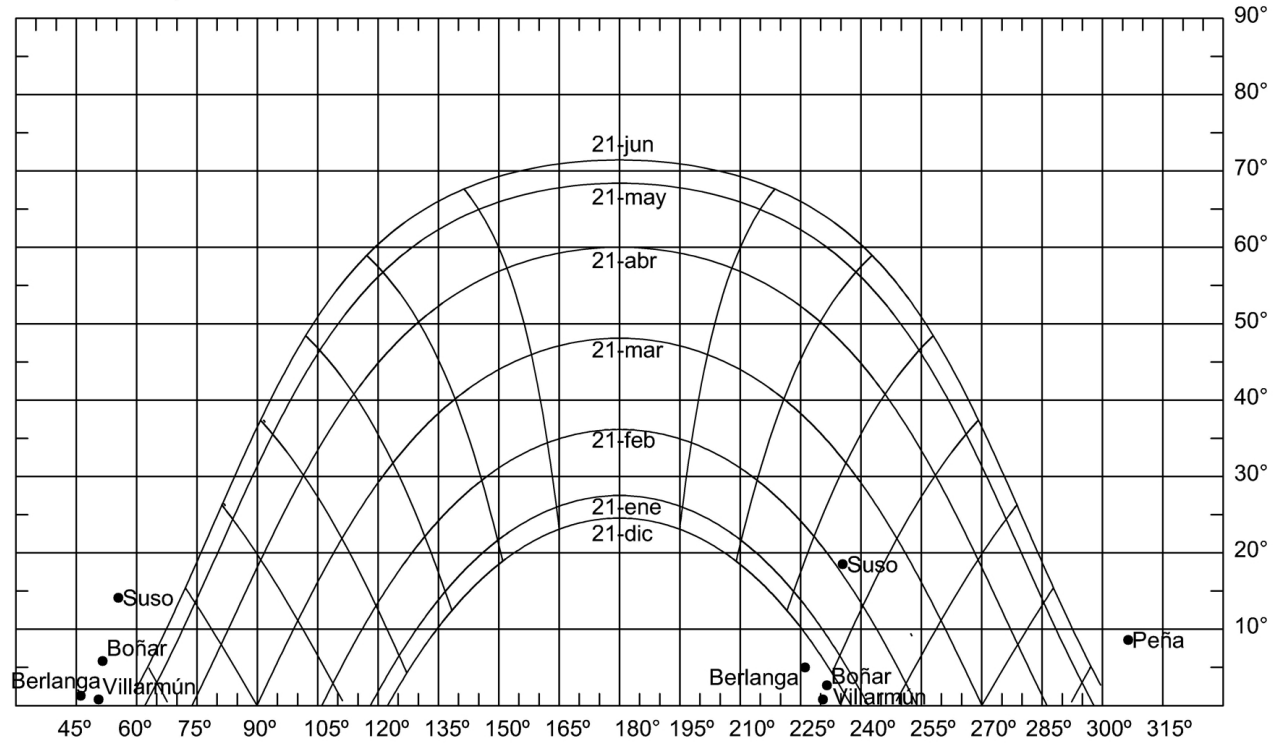

Figura 8. Posibles orientaciones al ocaso de iglesias mozárabes.

Esta posible orientación a poniente se ha podido observar en varias iglesias prerrománicas, como Lena o Samos, y en algunas románicas que probablemente se fundaron sobre otras previas, como Ripoll o Silió, por lo que este ejemplo es interesante como posible precedente. No se dispone de dato alguno que permita aventurar ninguna hipótesis que pueda explicar este hecho, pero sin duda permitiría explicar estas orientaciones. También una posible orientación al oeste permitiría situar en fechas más adecuadas del año los replanteos de las iglesias de invierno citadas, como Santa María de San Cristóbal de Boedo, Santa María de Retortillo, la Asunción de Camarzana de Tera, San Martín de Oliván y San Pelayo de Bodas.

La posible orientación a poniente de determinadas iglesias es una hipótesis plausible, pero es posible una explicación alternativa en el caso de las citadas, cuya orientación no corresponde con una posición posible del orto solar. Boñar y Villarmún están en el límite, por lo que es posible que se orientaran en una fecha próxima al solsticio de verano y simplemente hay un error en el replanteo. En los casos de San Millán de Suso y San Baudelio de Berlanga, la orientación se desvía tanto que no cabe la hipótesis del error. Sin embargo, en ambos casos, existen eremitorios a los que se adosó la iglesia. Su emplazamiento estaba prefijado y no se podía mover $y$, una vez fijado ese punto, la solución constructivamente sensata era edificar para-

32 Se hace constar que la carta solar corresponde a fechas gregorianas, por lo que sus datos deben ser corregidos a fechas julianas, las usadas por los constructores. Por otra parte las posibles variaciones para una diferencia de latitud de 1 a $2^{\circ}$ son muy poco significativas. 
lelamente a la ladera, escogiendo la orientación más próxima al este, dentro de lo factible, que es exactamente lo que se ha hecho. No tenemos ninguna prueba precisa, pero la coincidencia es notable, lo que podría ser un indicio de una posible aplicación de un criterio de tipo topográfico.

El caso más singular a efectos de orientación es la Santa María de Hérmedes de Cerrato. Es una pequeña ermita construida con muros de mampostería y sillarejo de aparejo muy basto. Las esquinas están algo mejor aparejadas con algunos sillares mejor tallados. Se compone de una sola nave rectangular con un ábside cuadrado con bóveda sobre pechinas, separado de la nave por un arco triunfal de herradura. Lo más notable de esta iglesia es su orientación, casi norte, $337,11^{\circ}$, absolutamente atípica. Esta ermita ha planteado muchas dudas, en especial por sus muros exteriores, probablemente mucho más modernos, al menos, en su parte visible. Esto ha hecho suponer que el arco triunfal, sin duda de trazado mozárabe, pudiera haber sido recolocado en una iglesia que se hubiera edificado posteriormente. Lo que ya parece muy improbable es que se haya trasladado la bóveda mozárabe sobre el ábside o se haya reconstruido posteriormente, lo que para este tipo de bóvedas es una obra complicada y cara. De haber tratado de trasladar el arco, lo más lógico es que la bóveda hubiera sido resuelta en el estilo de su época. Esta operación se hizo en la discutida restauración de San Cebrián del Mazote, pero está documentada. Por los escasos datos disponibles, esta ermita nunca ha sido objeto de grandes intervenciones. Dilucidar esta cuestión exigiría un estudio de la arqueología de la arquitectura, en este momento muy improbable.

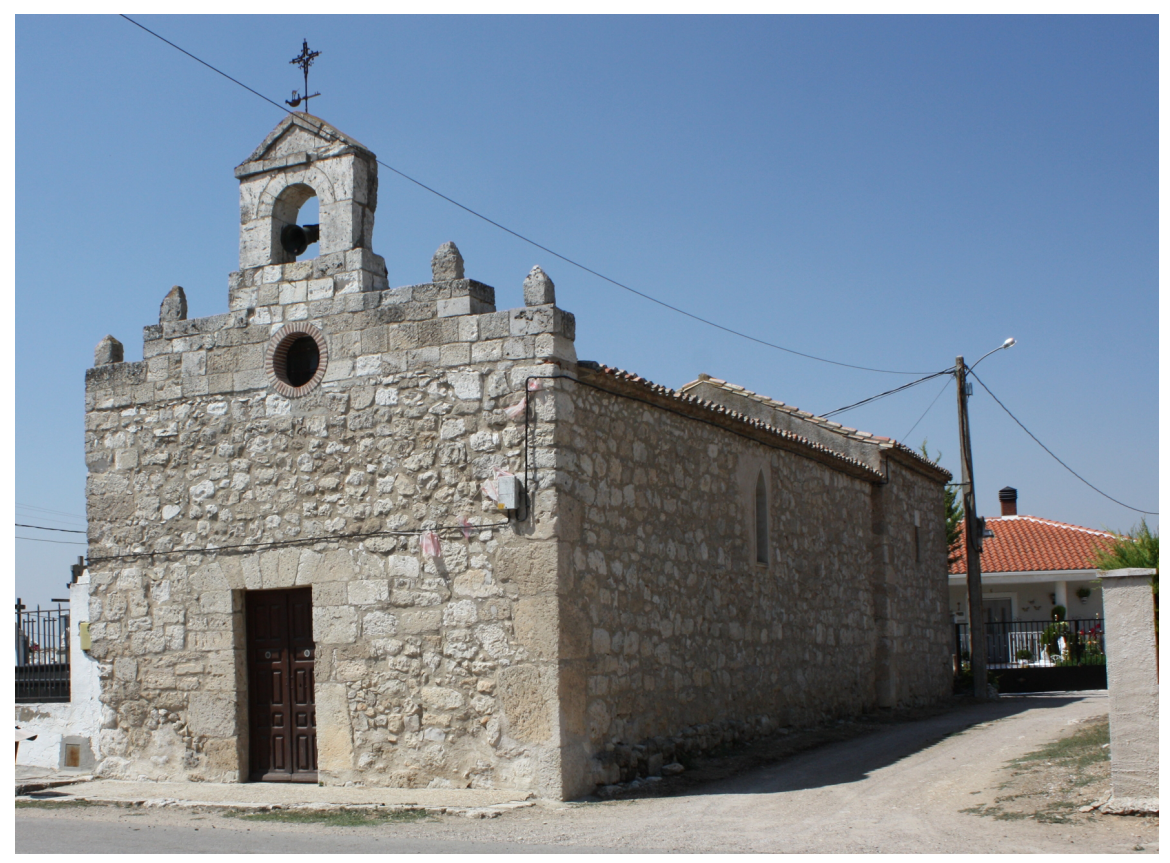

Figura 9. Hérmedes de Cerrato (Palencia).

No disponemos de ninguna documentación directa, pero si de algunos datos indirectos que pudieran servir de explicación. La Crónica najerense, al hablar de las 
campañas de Alfonso I, nos habla de una ataque a Saldaña y Simancas, que probablemente pasó por esta localidad o muy cerca ${ }^{33}$. La política de este rey consistió en despoblar la frontera del Duero, expulsando a los musulmanes y llevando a los cristianos a los reinos del norte. En el mismo párrafo de la Crónica najerense se expresa con claridad: "Mató con la espada a todos los árabes que encontró en las antedichas ciudades, y a los cristianos se los llevó consigo a la patria". Esta política de tierra quemada dejó despoblada la ribera norte del Duero, salvo algunas ciudades fuertemente fortificadas, lo que protegió a los reinos del norte de los ejércitos emirales primero y califales después. En siglos posteriores, la repoblación de esta zona fue uno de los motores del desarrollo de la arquitectura mozárabe.

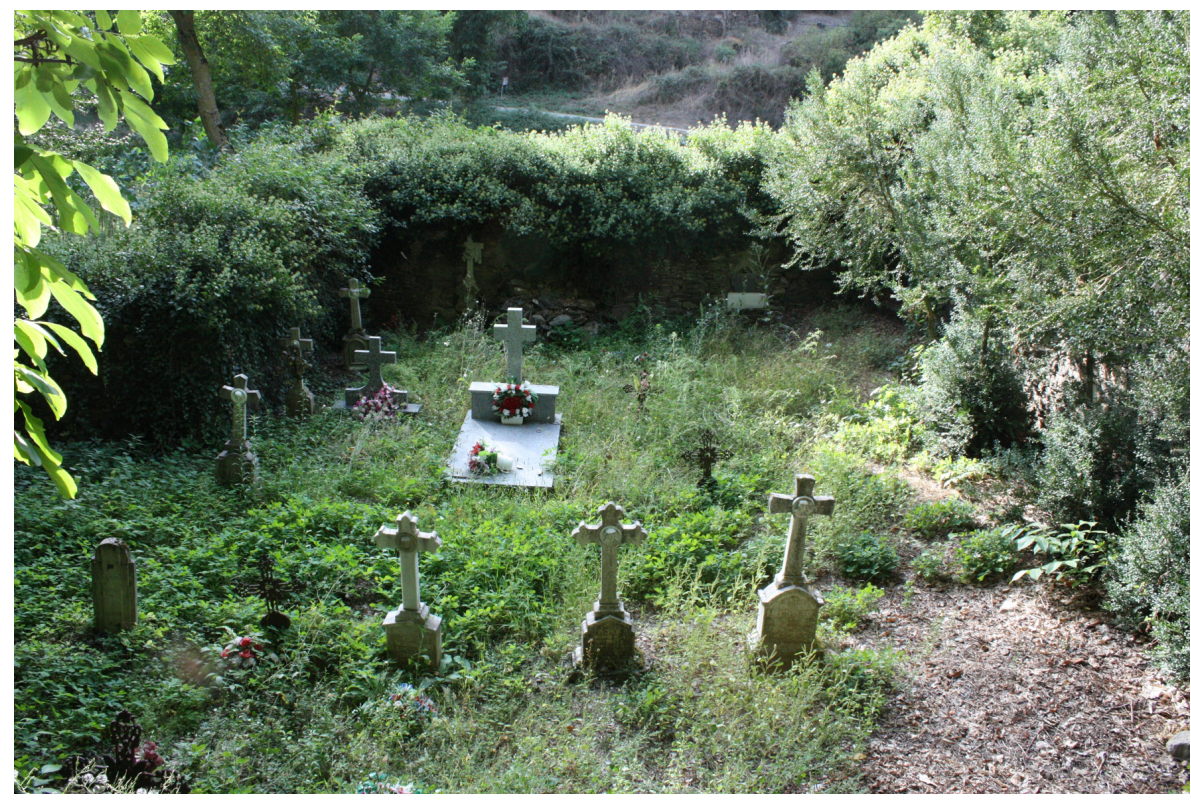

Figura 10. Valdueza (León).

Es muy probable que en esta localidad existiera una pequeña mezquita orientada hacia el sur, como era habitual en las mezquitas omeyas españolas. Con este criterio, la orientación de esa supuesta mezquita sería de $157,11^{\circ}$ y, a título de ejemplo, podemos señalar que la orientación de la mezquita de Córdoba es de $151,17^{\circ}$. Tras la sangrienta campaña de Alfonso I, la localidad debió quedar abandonada y, cuando se realizó la repoblación, algunos mozárabes pudieron decidir reaprovechar la nave de la mezquita, añadiendo un ábside mozárabe. Simplemente y para diferenciarse de los musulmanes, la orientaron al revés. Hemos podido comprobar que esto se realizó en algún otro caso documentado, como la iglesia de San Sebastián de Toledo, con una orientación de $343,10^{\circ}$ que está construida reaprovechando una mezquita (alDabbagin) orientada a $163,10^{\circ}$. Naturalmente es una simple hipótesis, sin confirmación documental, pero nos parece una interesante línea de trabajo. 
También es curioso el caso de la iglesia de San Clemente de Valdueza, próxima a las iglesias de Peñalba y Montes en la Tebaida Leonesa, que en este momento está totalmente arruinada y convertida en cementerio. El propio Gómez-Moreno observó unos detalles que hacen dudar de su orientación:

Su frente oriental ciérrase en dirección algo oblicua con pared llana, que no muestra apariencias de ser moderna, y enfrente hay una ventana abocinada y con arco de herradura, groseramente hecha, como todo. Será cosa del siglo X; pero ventanas así, en bajo, no eran costumbre a los pies de las iglesias, sino en su cabecera, de suerte que asalta la sospecha de si esta última iría dispuesta, contra la orientación de ritual, encarando su altar hacia poniente ${ }^{34}$.

Según nuestras medidas, la orientación a levante es de $94,4^{\circ}$, pero considerando la fortísima pendiente de $36,4^{\circ}$, supone una posición del orto solar a $61,59^{\circ}$ (17 de mayo y 18 de julio). Si consideramos la orientación a poniente, tendríamos $274,4^{\circ}$. En este caso, la pendiente es menor, lo que supone una posición del ocaso de $294,27^{\circ}$ ( 26 de enero y 6 de noviembre). Basados en criterios fundamentalmente arquitectónicos, coincidimos plenamente con la opinión de Gómez-Moreno, incluso con la desventaja de haber visto el edificio aún más deteriorado. Por otra parte, la orientación a poniente da una fecha, 6 de noviembre, próxima a la festividad de San Clemente, 23 de noviembre, que además coincide en todos los santorales consultados. Sin duda, las pruebas no son concluyentes, pero nos inclinamos por considerar que la iglesia estaba orientada a poniente y posiblemente siguiendo el criterio simbólico.

\section{Conclusiones}

Creemos que el estudio realizado permite llegar a unas conclusiones razonables, aunque sin duda hemos de admitir cierto grado de incertidumbre. Al igual que en iglesias construidas en otros períodos históricos, el criterio simbólico de orientación no es compatible con los resultados obtenidos para la mayoría de las iglesias mozárabes. Sin embargo, el número de coincidencias es considerablemente mayor en términos porcentuales de las observadas en las iglesias visigóticas, prerrománicas y románicas, por lo que no puede ser rechazado tajantemente. Es posible que en comunidades que provenían de una situación muy difícil para el desarrollo del culto, se hubiera acentuado el sentido simbólico del templo. En todo caso, tampoco la coincidencia es suficiente para suponer que fuera una postura unánime, ni siquiera mayoritaria.

El criterio topográfico no parece ser regla común en las iglesias mozárabes, en la que no se aprecia un particular interés en adaptarse al terreno o en buscar ubicaciones especiales. Sin embargo, hay dos notables casos, Berlanga y Suso, en los que los condicionantes simbólicos, pero a la vez topográficos del lugar, pueden explicar sus orientaciones atípicas. Por otra parte, existe una notable incidencia de orientaciones hacia un pico montañoso en las iglesias del Serrablo. Al no haber otro tipo de datos, únicamente podemos resaltar la coincidencia.

El criterio litúrgico podría ser aplicado a un porcentaje significativo de iglesias, pero para poder validarlo sería necesario conocer el año exacto del replanteo, puesto

34 Gómez-Moreno, Iglesias mozárabes, p 218. 
que la Pascua es una festividad variable. Solo es escasísimas ocasiones está documentado dicho año, como en el caso de la abadía de Limburg an der Haardt en 1030 en presencia del emperador Conrado II, que cita Nissen ${ }^{35}$. El criterio litúrgico puede plantearse en el terreno hipotético y de hecho su porcentaje es bastante alto, similar al que hemos encontrado en iglesias visigóticas y románicas y claramente superior a las prerrománicas. La pauta es bastante parecida a las iglesias visigóticas en las que las orientaciones predominantes corresponden al período pascual y posterior. Es una tendencia diferente a la de las iglesias románicas, cuyas orientaciones se concentran en la Pascua y el período inmediatamente anterior a ella. Entendemos que no hay razones suficientes para aceptarlo con claridad, aunque tampoco para rechazarlo de plano.

En opinión de los autores, el criterio más probable es el puramente constructivo, puesto que responde a un porcentaje muy significativo de las orientaciones medidas. Creemos que, en la mayoría de los casos, la iglesia fue orientada coincidiendo con el replanteo, por razones puramente funcionales. Probablemente se eligió un día seco y soleado que habría permitido fijar con facilidad la posición del orto solar y realizar cómodamente el replanteo. Ese día, en muchos casos, no respondió a ningún criterio ni simbólico ni litúrgico. Al igual que pasa en otros templos, como en las iglesias visigóticas, es probable que entre los comitentes de la obra hubiera personas con conocimientos astronómicos que hubieran permitido la fijación correcta del este geográfico y, sin embargo, no fue así. Las razones de esa desviación nos son desconocidas, pero el hecho objetivo es que las orientaciones medidas suponen posiciones del orto solar que cubren casi todas las fechas posibles del año, con una mayor frecuencia en primavera, que siempre fue el período habitual de comienzo de las obras. Hay documentación medieval, si bien posterior, que avala esta aseveración. De hecho, los contratos que menciona Hinton suelen fijar una fecha de primavera. Por ello, entendemos que la fecha más probable para el replanteo es un día de primavera o próximo a ella y en un día adecuado con el suelo seco y cielo despejado. Aún hoy en día sigue siendo lo razonable en la construcción tradicional. Al contrario de otros tipos de iglesias medievales como las visigóticas, prerrománicas o románicas en las que la probabilidad del uso de criterios simbólicos o litúrgicos es muy baja, en el caso de las iglesias mozárabes las coincidencias son mucho mayores. No son lo suficientemente relevantes como para fijar una pauta, pero deben considerarse como opciones probables a la hora de explicar algunos casos concretos.

A manera de resumen, entendemos que existen coincidencias que hacen pensar que todos los criterios citados se han podido usar en algunos casos y es probable que no se pueda hablar de un criterio único, sino de probabilidades. El criterio simbólico podría ser válido en un $18,5 \%$ de los casos, que es un porcentaje significativamente mayor que en otros estilos. El criterio litúrgico podría aplicarse a un $42,86 \%$ de los casos, pero con la grave incertidumbre del año de replanteo. Entendiendo que el período pascual es de cuatro semanas y considerando que la intencionalidad de la orientación podría referirse a la semana de pascua, sin fijar un día concreto, la posible coincidencia se produciría con una probabilidad de $10,71 \%$. En cuanto al criterio topográfico, al menos en dos casos muy notables, Berlanga y Suso, existen elementos previos altamente simbólicos que marcan el lugar y pueden definir la orientación por adaptación al terreno. También es notable la alta incidencia de orien-

35 Nissen, Orientation, Studien zur Geschichte der Religion, pp. 402. 
taciones hacia un pico montañoso en las iglesias del Serrablo. Con todo, creemos que el criterio aplicado en la mayoría de los casos fue el constructivo, con todas las salvedades citadas.

\section{Bibliografía}

Abrahamsen, Niels, "Evidence for church orientation by magnetic compass in twelfth-century Denmark". Archaeometry, 34/2 (1992), pp. 293-303. DOI: 10.1111/j.1475-4754.1992.tb00499.x.

Agustí y Casanovas, Jacinto y Voltes Bou, Pedro, Manual de cronología española y universal, Madrid, 1952.

Ali, Jason R. y Cunich, Peter "The Orientation of Churches: Some New Evidence", The Antiquaries Journal, 81, (2001), pp. 155-193. DOI: 10.1017/ S0003581500072188.

Azuar, Rafael, "De arqueología mozárabe”, Arqueología y Territorio Medieval 22, (2015), pp. 121-145. DOI: 10.17561/aytm.v22i0.2678.

- "De arqueología mozárabe II. De sus iglesias y documentos epigráficos". Arqueología y Territorio Medieval, 23, (2016), pp. 75-102. DOI 10.17561/aytm. v23i0.3186.

Bango Torviso, Isidro "Arquitectura de la décima centuria: ¿Repoblación o mozárabe?". Goya: Revista de Arte, 122 (1974), pp. 68-75.

Cave, Charles J.P., "The Orientation of Churches", The Antiquaries Journal, 30 (1950), pp. 47-51.

Crónica najerense, ed. de Juan A. Estévez Sola, Madrid, 2003.

Delgado Gómez, Jaime, 2006: "El porqué de la orientación de las iglesias", Lucensia, 16/33, pp. 347-356.

Duffett-Smith, Peter, Practical Astronomy with Your Calculator, Cambridge, 1988.

Gómez-Moreno, Manuel, Iglesias mozárabes. Arte español de los siglos IX a XI, Madrid, 1919.

González-García, Antonio C., "A Voyage of Christian Medieval Astronomy: Symbolic, Ritual and Political Orientation of Churches", en F. Pimenta, N. Ribeiro, F. Silva, N. Campion, A. Joaquinito y L. Tirapicos (eds.), Stars and Stones: Voyages in Archaeoastronomy and Cultural Astronomy. 19th Meeting of the European Society for Astronomy in Culture (Évora, Portugal, 19th-23rd September, 2011), Oxford, 2015, pp. 240-248.

- "La orientación de las iglesias prerrománicas de Galicia: análisis y resultados preliminares", Estudos do Quaternário, 12 (2015), pp. 133-142,

- y Belmonte, Juan A., "The Orientation of Pre-Romanesque Churches in the Iberian Peninsula", Nexus Network Journal, 17 (2015), pp. 353-377. DOI 10.1007/ s00004-014-0231-7.

Hinton, Ian, "Church Alignment and Patronal Saint's Days", The Antiquaries Journal, 86 (2006), pp. 206-226.

- Aspects of the Alignment and Location of Medieval Rural Churches, tesis doctoral inédita leída en University of East Anglia, 2010, disponible en https://ueaeprints. uea.ac.uk/20521.

Hoare, Peter G. y Sweet Caroline S., "The Orientation of Early Medieval Churches in England", Journal of Historical Geography, 26/2, (2000), pp. 162-173. 
Knapp, A. Bernard y Ashmore, Wendy, "Archaeological Landscapes: Constructed, Conceptualized, Ideational", en Wendy Ashmore y A. Bernard Knapp (eds) Archaeologies of Landscape: Contemporary Perspectives, Oxford, 1999, pp. 1-30.

Krautheimer, Richard, Arquitectura paleocristiana y bizantina, Madrid, 1984.

Laužikas, Rimvydas, "Church Orientations in Central and Eastern Europe", en Clive L. N. Ruggles (ed,), Handbook of Archaeoastronomy and Ethnoastronomy, Nueva York, 2015, pp. 1727-1732. DOI 10.1007/978-1-4614-6141-8.

Martínez Enamorado, Virgilio, "Sobre las 'cuidadas iglesias' de Ibn Hafsun: estudio de la basílica hallada en la ciudad de Bobastro (Ardales, Málaga)", Madrider Mitteilungen, 45 (2004) p. 507-531.

McCluskey, Stephen C., Astronomies and Cultures in Early Medieval Europe. Cambridge, 1998.

- "Orientation of Christian Churches", en Clive L. N. Ruggles (ed.), Handbook of Archaeoastronomy and Ethnoastronomy, Nueva York, 2015. DOI: 10.1007/9781-4614-6141-8 173.

Nissen, Heinrich, Orientation, Studien zur Geschichte der Religion, Berlín, 1906.

Pérez Valcárcel, Juan, "La orientación de las iglesias románicas del Camino de Santiago", en $2^{\circ}$ Congreso Nacional de Historia de la Construcción, La Coruña, 1998, pp. 391-396.

- y Pérez Palmero, Victoria, "El fenómeno de la luz equinoccial en Santa Marta de Tera", Brigecio. Revista de Estudios Benaventanos, 23 (2013), pp. 67-78.

Persson, Kjell, "The Orientation of Vendel Church", Journal of Nordic Archaeological Science, 14 (2004), pp. 139-141.

Rodríguez Arós, Ángel D., Blanco, Francisco y Muiños, María José, Trigonometría plana y esférica con aplicaciones a la navegación, Madrid, 2012.

Sassin Allen, Anne, "Church Orientation in the Landscape: a Perspective from Medieval Wales", Archaeological Journal, 173/1 (2016), pp. 154-187. DOI: 10.1080/00665983.2016.1110781.

Simonet, Francisco J., Santoral hispano-mozárabe escrito en 961 por Rabi Ben Zaid, obispo de Iliberis, Madrid, 1871.

Spinazzè, Eva, "The Alignment of Medieval Churches in Northern-Central Italy and in the Alps and the Path of Light Inside the Church on the Patron Saint's Day". Mediterranean Archaeology and Archaeometry, 16/4 (2016), pp. 455-463.

Tertuliano, Quinto Septimio Florente, Apologético a los gentiles, introducción, traducción y notas de Carmen Castillo García, Madrid, 2001.

Vives Castell, José, "Santoral visigodo en calendarios e inscripciones", Analecta Sacra Tarraconensia: Revista de Ciències Historicoeclesiàstiques, 14 (1941), pp. 31-58.

Vogel Cyrille, "Sol aequinoctialis. Problèmes et technique de l'orientation dans le culte chrétien", Revue des Sciences Religieuses, 36/3-4 (1962), Archéologie paléochrétienne et culte chrétien, pp. 175-211. DOI: 10.3406/rscir.1962.2332. 